\section{Arkivoc

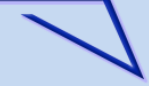

Archive for

Organic Chemistry
The Free Internet Journal

for Organic Chemistry
Paper

Arkivoc 2017, part ii, 407-420

\title{
Synthesis and spectroscopic characterization of double chained and sulfurated derivatives of L-ascorbic acid
}

\author{
Damiano Tanini, Marianna Gori, Francesco Bicocchi, Moira Ambrosi, Pierandrea Lo Nostro, \\ and Antonella Capperucci*
}

Dipartimento di Chimica "Ugo Schiff", Università di Firenze, Via della Lastruccia 3-13, 50019 Sesto Fiorentino, Italy

E-mail: antonella.capperucci@unifi.it

Dedicated to Prof. Jacek Młochowski on the occasion of his $80^{\text {th }}$ anniversary

Received 07-04-2016

Accepted 09-23-2016

Published on line 12-04-2016

\section{Abstract}

Lipophilic saturated and unsaturated L-ascorbyl 5,6-O-diesters from fatty acids are prepared and fully characterized through NMR and MS spectra. Derivatives with different sulfurated moieties are obtained as well through thio-Michael addition of thiols on ascorbyl acrylates. The new amphiphilic structures exhibit very high antioxidant activity using the DPPH assay.

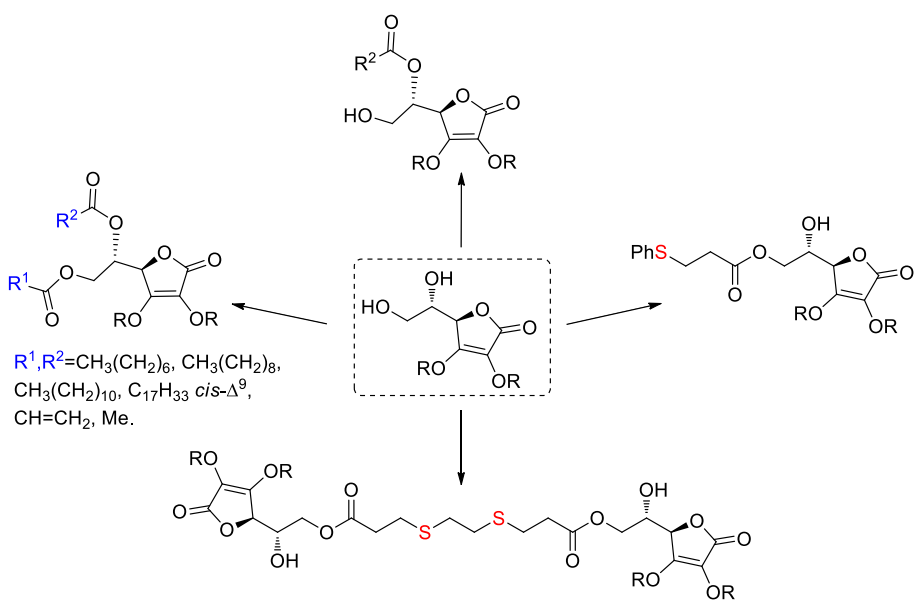

Keywords: L-Ascorbic acid, fatty acids, antioxidant, sulfurated ascorbyl esters, thio-Michael addition 


\section{Introduction}

Antioxidants are widely used in different fields, for example the food, cosmetic and pharmaceutical industries. Our interest in the chemistry of sulfurated and selenated compounds led us to develop an access to organochalcogen derivatives, such as selenides, diselenides and heterocyclic compounds, ${ }^{1-6}$ which evidenced interesting antioxidant activity. The chemistry of organosulfur, and more recently of organoselenium compounds has in fact experienced a strong development. ${ }^{7,8}$ Such derivatives are used as reagents in organic synthesis and, due to their interest from the biological point of view, in the preparation of molecules with biological activity. Recently we were involved in the study of modifications of natural products, with the aim to introduce on the same molecular skeleton sulfurated or selelenated moieties, which could possess a synergistic effect together with the natural core. We found a convenient method for the synthesis of selenium-derivatives of resveratrol as antioxidants and free radical scavengers. ${ }^{9}$ These structures evidenced antioxidant activity with Trolox-like capacity.

In this context considerable interest has also been devoted to the study of liposoluble ester derivatives of L-ascorbic acid (L-ASC), which is a well known, potent and versatile antioxidant. Their physico-chemical properties have been extensively investigated ${ }^{10-17}$ in particular their phase behavior in the solid state and in aqueous dispersions. The amphiphilic derivatives of ascorbic acid fully retain the antioxidant power of the parent molecule, and produce nanostructured self-assembled systems both in aqueous and non aqueous media, and therefore they can act as carriers for important hydrophobic molecules such as drugs and nutraceutics.

Certainly, a very large number of contributions have been reported on vitamin $C$, most of them dealing with the elaboration at positions 2,3 or 6 , depending on the application needs. ${ }^{18-22}$ Generally 6 -O-ascorbyl esters are obtained via different methods, by reacting vitamin $\mathrm{C}$ and fatty acids under catalysis of lipases ${ }^{20}$ or in concentrated sulfuric acid. ${ }^{23}$

Here we report the preparation and full characterization of lipophilic saturated and unsaturated 5,6-Odiesters of L-ASC, as well as of sulphur-containing 6-O-ascorbyl alkanoates. Preliminary evaluation of their antioxidant properties will also be described.

\section{Results and Discussion}

To the best of our knowledge, few examples of synthesis of 5,6-diesters of L-ASC have been reported. Nevertheless, a very large number of examples of esterification at C-6 with palmitoyl chloride were described in a patent, bearing different substituents and protecting groups on selected hydroxyls. ${ }^{24} \mathrm{Chemical}$ modifications of vitamin $C$ are often limited by its intrinsic instability. Typically, together with the methods cited above, the chemical synthesis of ascorbyl esters is carried out by selective protection and deprotection of functional groups. Several steps are required to obtain the target compounds. Usually the first step involves the protection of hydroxyl groups on C- 6 and C-5 as an acetonide, followed by treatment of the enol hydroxyls with benzyl bromide. ${ }^{21,25}$ Nevertheless, on the basis of results recently reported ${ }^{26}$ and of our own results in separate experiments, we observed a high, selective reactivity of the ene-diol portion with benzyl bromide by comparison with the alcohols at the 5 and 6 positions. This allowed us to treat L-ASC 1 with $\mathrm{BnBr}$ and $\mathrm{K}_{2} \mathrm{CO}_{3}$ in 
THF/DMSO (Scheme 1) directly, thus avoiding preliminary protection as 5,6-O-isopropylidene derivative and the following deprotection step.

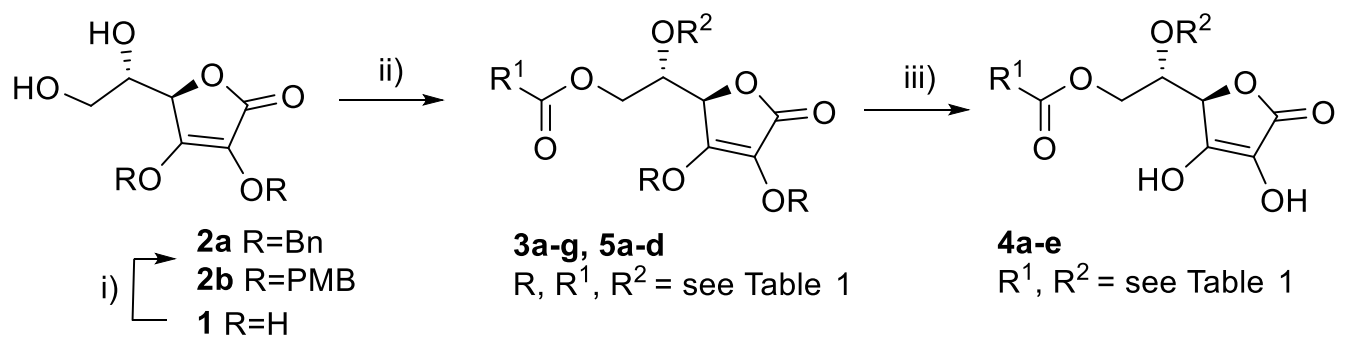

Scheme 1. (i) $\mathrm{RBr} / \mathrm{K}_{2} \mathrm{CO}_{3}, \mathrm{THF} / \mathrm{DMSO}, 50{ }^{\circ} \mathrm{C}$, $3 \mathrm{~h}$; (ii) acyl chlorides, 4-DMAP/DCC or Et ${ }_{3} \mathrm{~N}$ (see experimental); (iii) $\mathrm{H}_{2}, \mathrm{Pd} / \mathrm{C}$ or $\mathrm{SnCl}_{4} / \mathrm{PhSH}$ (see experimental).

The dibenzyl ether $2 \mathrm{a}$ was then treated with saturated fatty acid derivatives. Thus acyl chlorides of octanoic (caprylic, $C_{8}$ ), decanoic (capric, $C_{10}$ ) and dodecanoic (lauric, $C_{12}$ ) acid were reacted in the presence of DMAP/DCC in acetonitrile (Scheme 1). L-Ascorbyl 5,6-O-dialkanoates 3a-c were obtained in high yields (Table 1, entries 1-3). Cleavage with $\mathrm{H}_{2}, \mathrm{Pd} / \mathrm{C}$ led to the formation in quantitave yield of the previously unreported 2,3-(OH) 2-5,6-diesters 4a-c, which were fully characterized by means of NMR and MS spectra.

Table 1. Synthesis of saturated and unsaturated L-ASC esters

\begin{tabular}{|c|c|c|c|c|c|}
\hline Entry & $\mathrm{R}$ & $\mathrm{R}^{1}$ & $R^{2}$ & $\begin{array}{l}\text { Products } \\
(\text { Yield \%) }\end{array}$ & $\begin{array}{r}\text { Products } 4 \\
(\text { Yield \%) } \\
\end{array}$ \\
\hline 1 & $\mathrm{Bn}$ & $\mathrm{CH}_{3}\left(\mathrm{CH}_{2}\right)_{6}$ & $\mathrm{CO}\left(\mathrm{CH}_{2}\right)_{6} \mathrm{CH}_{3}$ & 3a (90) & $4 a(>98)$ \\
\hline 2 & $\mathrm{Bn}$ & $\mathrm{CH}_{3}\left(\mathrm{CH}_{2}\right)_{8}$ & $\mathrm{CO}\left(\mathrm{CH}_{2}\right)_{8} \mathrm{CH}_{3}$ & $3 b(88)$ & $4 b(>98)$ \\
\hline 3 & $\mathrm{Bn}$ & $\mathrm{CH}_{3}\left(\mathrm{CH}_{2}\right)_{10}$ & $\mathrm{CO}\left(\mathrm{CH}_{2}\right)_{10} \mathrm{CH}_{3}$ & $3 c(91)$ & $4 c(>98)$ \\
\hline 4 & $\mathrm{Bn}$ & $\mathrm{CH}_{3}\left(\mathrm{CH}_{2}\right)_{10}$ & $\mathrm{COCH}_{3}$ & $3 d(63)^{b}$ & $\mathbf{4 d}(>98)$ \\
\hline 5 & $\mathrm{Bn}$ & $\mathrm{C}_{17} \mathrm{H}_{33}$ cis- $\Delta^{9}$ & $(\mathrm{CO}) \mathrm{C}_{17} \mathrm{H}_{33}$ cis- $\Delta^{9}$ & 3e (78) & - \\
\hline 6 & PMB & $\mathrm{C}_{17} \mathrm{H}_{33} c i s-\Delta^{9}$ & $(\mathrm{CO}) \mathrm{C}_{17} \mathrm{H}_{33}$ cis- $\Delta^{9}$ & $3 f(83)$ & $4 e(34)$ \\
\hline 7 & $\mathrm{Bn}$ & $\mathrm{CH}=\mathrm{CH}_{2}$ & $(\mathrm{CO}) \mathrm{CHCH}_{2}$ & $3 g(75)$ & - \\
\hline 8 & PMB & $\mathrm{C}_{17} \mathrm{H}_{33} c i s-\Delta^{9}$ & $\mathrm{H}$ & $5 a(48)$ & - \\
\hline 9 & $\mathrm{Bn}$ & $\mathrm{CH}=\mathrm{CH}_{2}$ & $\mathrm{H}$ & $5 b(53)^{c}$ & - \\
\hline 10 & PMB & $\mathrm{CH}=\mathrm{CH}_{2}$ & $\mathrm{H}$ & $5 c(48)^{c}$ & - \\
\hline 11 & $\mathrm{Bn}$ & $\mathrm{CH}_{3}\left(\mathrm{CH}_{2}\right)_{10}$ & $\mathrm{H}$ & $5 d(68)$ & - \\
\hline
\end{tabular}

a Isolated yields. ${ }^{b}$ Global yield over two steps. ${ }^{c} 10 \%$ of diester was also formed.

In order to synthesize derivatives with different acyl chain lengths, for evaluating the influence on physicochemical properties, compound 3d (Table 1, entry 4) was prepared in two steps from lauroyl- and acetylchlorides (see experimental). The final deprotection afforded the mixed diester $\mathbf{4 d}$ (Scheme 1). All the diesters were obtained with high selectivity (>99\%), no trace of monoesters being observed.

Our interest also focused in the preparation of ascorbyl oleates. These compounds bear, on the same molecular skeleton, both oleic and ascorbic acid residues, that each possess antioxidant properties. However, the synthesis was not straightforward, the products being rather sensitive compounds. This makes ascorbyl oleate difficult to produce with traditional chemical methods. To the best of our knowledge only one example 
has been described for ascorbyl 6-monooleate, ${ }^{27}$ while enzymatic methodologies are generally reported. ${ }^{28-34}$ The search for alternative methods is therefore a challenge to access these molecules.

Following a similar approach, oleoyl chloride was reacted with 2 a leading to the formation of 2,3-Odibenzyl-5,6-O-ascorbyl dioleate 3 e in good yield (Scheme 1 and Table 1, entry 5). For the cleavage of benzylic ethers, the incompatibility of $\mathrm{H}_{2}, \mathrm{Pd} / \mathrm{C}$ system with the double bonds prompted us to seek alternative, mild methods. Unfortunately, treatment with CSI (chlorosulfonyl isocyanate) ${ }^{35}$ did not afford the desired compound, but a complex mixture of products was formed. Thus, a different protecting group - $p$ methoxybenzyl (PMB) - was chosen. PMB can be more easily cleaved with numerous reagents, avoiding strong conditions. ${ }^{36,37} \mathrm{PMB}$-protected ascorbic acid $\mathbf{2} \mathbf{b}$ was prepared under the same conditions by direct treatment of $\mathrm{L}-\mathrm{ASC}$ with $\mathrm{PMBBr} / \mathrm{K}_{2} \mathrm{CO}_{3}$ (Scheme 1). Subsequent reaction with oleyl chloride afforded 2,3-O- $p$ methoxybenzyl-5,6-O-ascorbyl dioleate $3 f$ (Scheme 1 and Table 1, entry 6) or 2,3-O-p-methoxybenzyl-6-Oascorbyl oleate $5 a$ (Table 1, entry 8), depending on the reaction conditions (treatment with DMAP/DCC for diester, with $\mathrm{Et}_{3} \mathrm{~N}$ for monoester). Efficient cleavage of $3 \mathrm{f}$ with $\mathrm{SnCl}_{4} /$ thiophenol ${ }^{37}$ at $-78{ }^{\circ} \mathrm{C}$ led to the isolation of the 2,3-deprotected ascorbyl dioleate $4 \mathbf{e}$, while reaction on 5 a allowed cleavage of only one PMBO-group (most likely on C-2). Further experiments are under investigation to obtain complete deprotection at both positions. Nonetheless, the result obtained is rather interesting, providing new ascorbyl oleates through a convenient, alternative access.

As a further step, we decided to evaluate the esterification on the secondary $\mathrm{OH}$ at position 5 , retaining a free hydroxyl at C-6. To achieve this, a preliminary protection of $6-\mathrm{OH}$ with trityl chloride was carried out on $\mathbf{2 a}$ and $\mathbf{2} \mathbf{b}$ following literature procedures (Scheme 2 ). ${ }^{25}$

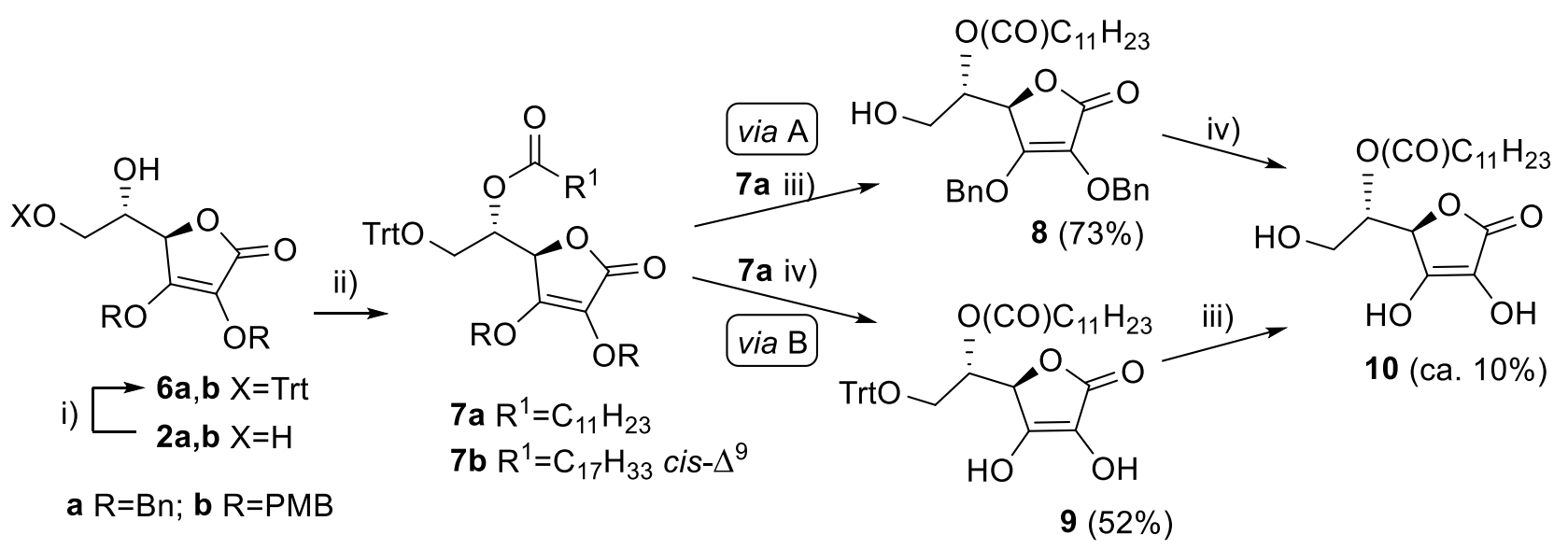

Scheme 2. (i) Trityl chloride/Et ${ }_{3} \mathrm{~N}$; (ii) $\mathrm{R}^{1} \mathrm{COCl} / \mathrm{DMAP} / \mathrm{DCC}$; (iii) $\mathrm{BF}_{3} \cdot \mathrm{Et}_{2} \mathrm{O}$; iv) $\mathrm{H}_{2}, \mathrm{Pd} / \mathrm{C}$.

After purification, the so obtained products $6 \mathbf{a}, \mathbf{b}$ were reacted respectively with lauroyl chloride and oleoyl chloride in the presence of DMAP/DCC, leading to $\mathbf{7 a}$ and $\mathbf{7 b}$ in good yields (Scheme 2). Deprotection of 6-OTrt under acidic conditions ${ }^{38}$ was performed by treatment of 7 a with $\mathrm{BF}_{3}$ etherate, leading to 8 (Scheme 2, via A). When reductive cleavage was carried out to obtain complete deprotection on $\mathrm{C}-2 / \mathrm{C}-3$, a very complex mixture was observed, and the desired product 10 was present in only very low yield $(<10 \%)$.

In order to seek better conditions, we firstly considered deprotection of benzyl groups in $7 \mathrm{a}$ with $\mathrm{H}_{2} / \mathrm{Pd} / \mathrm{C}$ (Scheme 2, via B). Compound 9 was isolated, and then treated under acidic conditions $\left(\mathrm{BF}_{3}\right)$ to remove the 
trityl group. Unfortunately, through this sequence, compound $\mathbf{1 0}$ was also formed in comparable yields (ca. $10 \%)$, and optimization of this step is surely required.


Scheme 3. (i) $\mathrm{PhSH}$, Neutral $\mathrm{Al}_{2} \mathrm{O}_{3}$; (ii) $\mathrm{HSCH}_{2} \mathrm{CH}_{2} \mathrm{SH}$, neutral $\mathrm{Al}_{2} \mathrm{O}_{3}$ (see experimental).

Nevertheless, compound $\mathbf{9}$ is interesting, in so far as the enediol moiety, responsible of the antioxidant activity of vitamin $\mathrm{C}$, is still present in this molecule.

Finally, to introduce a sulfurated or selenated group on the skeleton of vitamin $C$, we prepared ascorbyl 5,6-O-diacrylate $\mathbf{3 g}$ and 6-O-acrylates $\mathbf{5 b},{ }^{24} \mathbf{5 c}$ (Scheme 1 and Table 1 , entries 3, 9 and 10 ). Then $\mathbf{5 b}, \mathbf{5 c}$ and $\mathbf{3 g}$ were reacted respectively with one or two equivalents of a sulfur nucleophile (PhSH) in the presence of $\mathrm{Al}_{2} \mathrm{O}_{3}{ }^{39}$ (Scheme 3). Under these conditions compounds 11a, 11b and 12 were isolated, arising from a thio-Michael addition to the enones.

Even more interesting was the reaction of $\mathbf{5 b}$ with 1,2-ethanedithiol, providing the synthesis of the diascorbyl derivative 13, containing a sulfurated linker between the two vitamin C moieties (Scheme 3). It will be of interest to investigate the characteristics of such derivatives, including chains of different length, to compare their properties with the behaviour of bolaform surfactants studied by some of us. ${ }^{12}$ Search for conditions to deprotect 2,3-positions is currently under study in our laboratory.

Finally, in order to preliminary evaluate whether the antioxidant capacity was maintained for these new amphiphilic structures, they were tested through the DPPH assay, ${ }^{40}$ which provides an easy and rapid way to assess antioxidant potency. Substances $4 a-e$ and 9 exhibited a very high ability to act as free radical scavengers, in fact they instantaneously reacted with DPPH.

\section{Conclusions}

Different saturated and unsaturated ascorbyl derivatives, including novel sulfurated compounds, were prepared from fatty acids, and characterized by mono- and bidimensional NMR experiments. Their antioxidant activity was determined with the DPPH assay.

\section{Experimental Section}


General. NMR spectra were recorded in $\mathrm{CDCl}_{3}$ with Varian Gemini 200, Mercury 400, Inova 400 and Bruker 400 spectrometers operating at 200 and $400 \mathrm{MHz}\left({ }^{1} \mathrm{H}\right), 50$ and $100 \mathrm{MHz}\left({ }^{13} \mathrm{C}\right)$. NMR signals were referenced to non deuterated residual solvent signals of deuterochloroform (7.26 ppm for ${ }^{1} \mathrm{H}, 77.0 \mathrm{ppm}$ for $\left.{ }^{13} \mathrm{C}\right)$. Mass spectra (MS) were obtained by ESI. IR spectra were recorded on a Perkin Elmer Instrument (FT-IR). Only selected absorptions are reported, in wavenumbers $\left(\mathrm{cm}^{-1}\right)$. Solvents were dried using a solvent purification system (Pure-Solv ${ }^{\mathrm{TM}}$ ). Flash column chromatography was performed using silica gel (230-400 mesh). Where not specified, products were commercially available or obtained through reported procedures. 4Methoxybenzyl bromide (PMBBr) was synthesised from (4-methoxyphenyl)methanol (PMBOH) upon treatment with phosphorus tribromide. ${ }^{41} \mathrm{DPPH}$ assay was performed according to the literature, ${ }^{40}$ by measuring the adsorbance decreasing of a DPPH methanolic solution $\left(10^{-4} \mathrm{M}\right)$ treated with an equimolar amount of ascorbic acid derivatives (4 a-e, 9).

\section{General procedure for the protection of L-ascorbic acid as 2,3-dibenzyl- or 2,3-bis(p-methoxybenzyl)-ethers (GP1) 25}

Benzyl bromide or $p$-methoxybenzyl bromide (2.4 eq) was added to a suspension of L-ascorbic acid (1.0 eq) and $\mathrm{K}_{2} \mathrm{CO}_{3}(3.0 \mathrm{eq})$ in DMSO/THF (2:1 vol) and the reaction was stirred at $50{ }^{\circ} \mathrm{C}$ for $3 \mathrm{~h}$. Afterwards, the mixture was filtered through a Celite pad and the organic phase was extracted with EtOAc and washed with brine and $\mathrm{H}_{2} \mathrm{O}$. The organic layer was dried on $\mathrm{Na}_{2} \mathrm{SO}_{4}$, filtered and the solvent was removed under reduced pressure. The crude material was purified by flash chromatography (petroleum ether/EtOAc).

(R)-5-((S)-1,2-Dihydroxyethyl)-3,4-bis((4-methoxybenzyl)oxy)furan-2(5H)-one (2b). Following the general procedure, $1.8 \mathrm{~g}$ of L-ascorbic acid and $4.94 \mathrm{~g}$ of $p$-methoxybenzyl bromide gave, after purification, $2 \mathrm{~b}$ (1.91 g, $45 \%)$ as yellowish oil. ${ }^{1} \mathrm{H} N M R\left(400 \mathrm{MHz} \mathrm{CDCl}_{3}\right): \delta(\mathrm{ppm}) 3.80(\mathrm{~s}, 3 \mathrm{H}), 3.81(\mathrm{~s}, 3 \mathrm{H}), 3.71-3.81(\mathrm{~m}, 2 \mathrm{H}), 3.85-3.90$ $(\mathrm{m}, 1 \mathrm{H}), 4.64(\mathrm{~d}, J 2.4 \mathrm{~Hz}, 1 \mathrm{H}, \mathrm{CHO}), 5.06(\mathrm{ap} \mathrm{s}, 2 \mathrm{H}), 5.07(\mathrm{~d}, J 11.2 \mathrm{~Hz}, 1 \mathrm{H}), 5.12(\mathrm{~d}, J 11.2 \mathrm{~Hz}, 1 \mathrm{H}), 6.86$ (ap d, Is $8.6 \mathrm{~Hz}, 2 \mathrm{H}$ ), 6.91 (ap d, Is $8.6 \mathrm{~Hz}, 2 \mathrm{H}$ ), 7.15 (ap d, Is $8.6 \mathrm{~Hz}, 2 \mathrm{H}$ ), 7.33 (ap d, Is $8.6 \mathrm{~Hz}, 2 \mathrm{H}) .{ }^{13} \mathrm{C} \mathrm{NMR}(100 \mathrm{MHz}$, $\left.\mathrm{CDCl}_{3}\right): \delta$ (ppm) 55.3, 63.3, 69.9, 73.4, 73.7, 76.1, 113.9, 114.0, 120.8, 127.3, 128.1, 129.9, 130.9, 157.6, 159.9, 160.0, 170.1. Elemental Analysis: $\mathrm{C}_{22} \mathrm{H}_{24} \mathrm{O}_{8} \mathrm{C} 63.45 \%, \mathrm{H} 5.81 \%$. Found: $\mathrm{C} 63.22 \%, \mathrm{H} 5.83 \%$.

General procedure for the synthesis of ascorbyl 5-0-,6-O-dialkanoates protected as 2,3-dibenzyl ethers or 2,3-bis((4-methoxybenzyl) ethers. (GP2). 4-DMAP (12 mmol, 3 eq.) and DCC (12 mmol, 3 eq.) were added to a stirred solution of $\mathbf{2} \mathbf{a}$ or $\mathbf{2} \mathbf{b}(4 \mathrm{mmol}, 1$ eq.) in $\mathrm{MeCN}(40 \mathrm{~mL})$ under inert atmosphere at rt. Then the alkanoyl chloride $(9.2 \mathrm{mmol}, 2.3 \mathrm{eq}$.) was slowly added and the mixture was stirred for $12 \mathrm{~h}$. Afterwards the solvent was removed under reduced pressure and the crude material purified by flash chromatography (petroleum ether/EtOAc 8:1).

(S)-1-((R)-3,4-Bis(benzyloxy)-5-oxo-2,5-dihydrofuran-2-yl)ethane-1,2-diyl dioctanoate (3a). Following the general procedure GP2, 2a (1.0 g, $2.80 \mathrm{mmol})$ and octanoyl chloride $(1.043 \mathrm{~g}, 6.44 \mathrm{mmol})$ gave, after flash chromatography, $3 \mathbf{a}(1.53 \mathrm{~g}, 90 \%)$ as a colourless oil. ${ }^{1} \mathrm{H} \mathrm{NMR}\left(200 \mathrm{MHz}_{\mathrm{CDCl}}\right): \delta(\mathrm{ppm})$ 0.82-0.89 (m, 6H), 1.18-1.27 (m, 16H), 1.43-1.54 (m, 4H), 2.15-2.33 (m, 4H), 4.22 (dd, J 7.4, $\left.11.6 \mathrm{~Hz}, 1 \mathrm{H}, \mathrm{CH}_{\mathrm{a}} \mathrm{H}_{\mathrm{b}} \mathrm{O}\right), 4.33$ (dd, J 5.6, $11.6 \mathrm{~Hz}, 1 \mathrm{H}, \mathrm{CH}_{\mathrm{a}} \mathrm{H}_{\mathrm{b}} \mathrm{O}$ ), 4.80 (d, J $2.2 \mathrm{~Hz}, 1 \mathrm{H}, \mathrm{CHO}$ ), 5.10 (ap. s, 2H, $\mathrm{CH}_{2} \mathrm{Ph}$ ), 5.15 (ap. s, 2H, CH $2 \mathrm{Ph}$ ), 5.34-5.42 (m, $\left.1 \mathrm{H}, \mathrm{CHCH}_{2}\right), 7.19-7.25(\mathrm{~m}, 4 \mathrm{H}), 7.27-7.42(\mathrm{~m}, 6 \mathrm{H}) .{ }^{13} \mathrm{C} \mathrm{NMR}\left(50 \mathrm{MHz}, \mathrm{CDCl}_{3}\right): \delta(\mathrm{ppm}) 13.9,22.5,24.6,24.7$, 24.8, 28.7, 28.8, 28.9, 31.5, 33.8, 61.8, 67.3, 73.5, 73.6, 121.2, 127.9, 128.0, 128.6, 128.7, 128.8, 128.9, 129.0, 135.0, 135.9, 154.9, 168.3, 172.1, 172.8. MS (ESI positive) $\mathrm{m} / \mathrm{z}(\%): 632[\mathrm{M}+\mathrm{Na}]^{+},(100)$. Elemental Analysis: $\mathrm{C}_{36} \mathrm{H}_{48} \mathrm{O}_{8} \mathrm{C} 71.03 \%, \mathrm{H} 7.95 \%$. Found: C 70.82\%, H 7.98\%. 
(S)-1-((R)-3,4-Bis(benzyloxy)-5-oxo-2,5-dihydrofuran-2-yl)ethane-1,2-diyl didecanoate (3b). Following the general procedure GP2, 2a $(1.0 \mathrm{~g}, 2.80 \mathrm{mmol})$ and decanoyl chloride $(1.22 \mathrm{~g}, 6.44 \mathrm{mmol})$ gave, after flash chromatography, $3 \mathrm{~b}(1.64 \mathrm{~g}, 88 \%)$ as a colourless oil. ${ }^{1} \mathrm{H}$ NMR $\left(400 \mathrm{MHz}, \mathrm{CDCl}_{3}\right): \delta(\mathrm{ppm}) 0.84-0.90(\mathrm{~m}, 6 \mathrm{H})$, $1.25(\mathrm{~m}, 24 \mathrm{H}), 1.52-1.66(\mathrm{~m}, 4 \mathrm{H}), 2.19(\mathrm{dt}, J 2.2,7.1 \mathrm{~Hz}, 2 \mathrm{H}), 2.22-2.33(\mathrm{~m}, 2 \mathrm{H}), 4.22(\mathrm{dd}, J 7.0,11.7 \mathrm{~Hz}, 1 \mathrm{H}$, $\mathrm{CH}_{\mathrm{a}} \mathrm{H}_{\mathrm{b}} \mathrm{O}$ ), 4.32 (dd, J 5.9, $11.7 \mathrm{~Hz}, 1 \mathrm{H}, \mathrm{CH}_{\mathrm{a}} \mathrm{H}_{\mathrm{b}} \mathrm{O}$ ), 4.80 (d, J2.0 Hz, 1H, CCHO), 5.10 (ap s, 2H, CH $\mathrm{Ph}$ ), 5.14 (ap s, $\left.2 \mathrm{H}, \mathrm{CH}_{2} \mathrm{Ph}\right), 5.35-5.39\left(\mathrm{~m}, 1 \mathrm{H}, \mathrm{CHCH}_{2}\right), 7.21-7.23(\mathrm{~m}, 2 \mathrm{H}), 7.33-7.39(\mathrm{~m}, 8 \mathrm{H}) .{ }^{13} \mathrm{C} \mathrm{NMR}\left(50 \mathrm{MHz}, \mathrm{CDCl}_{3}\right): \delta(\mathrm{ppm})$ 13.7, 22.3, 24.3, 24.5, 24.7, 25.2, 28.8, 28.9, 29.1, 31.6, 33.6, 34.6, 55.3, 61.6, 67.2, 73.3, 73.5, 121.2, 127.7, 128.3, 128.4, 134.9, 135.7, 154.7, 168.1, 171.7, 172.5. MS (ESI positive) $m / z$ (\%): 687 [M+Na] $]^{+}$, (100). Elemental Analysis: $\mathrm{C}_{40} \mathrm{H}_{56} \mathrm{O}_{8} \mathrm{C} 72.26 \%$, $\mathrm{H} 8.49 \%$. Found: C 72.01\%, $\mathrm{H} 8.52 \%$.

(S)-1-((R)-3,4-Bis(benzyloxy)-5-oxo-2,5-dihydrofuran-2-yl)ethane-1,2-diyl didodecanoate (3c). Following the general procedure GP2, 2a (1.41 g, $3.95 \mathrm{mmol})$ and dodecanoyl chloride $(1.981 \mathrm{~g}, 9.1 \mathrm{mmol})$ gave, after flash chromatography, $3 \mathrm{c}(2.59 \mathrm{~g}, 91 \%)$ as a colourless oil. ${ }^{1} \mathrm{H} \mathrm{NMR}\left(200 \mathrm{MHz}, \mathrm{CDCl}_{3}\right): \delta(\mathrm{ppm}) 0.84-0.90(\mathrm{~m}, 6 \mathrm{H})$, $1.24(\mathrm{~m}, 32 \mathrm{H}), 1.48-1.53(\mathrm{~m}, 4 \mathrm{H}), 2.14-2.26(\mathrm{~m}, 4 \mathrm{H}), 3.83$ (ap d, Is $\left.6.2 \mathrm{~Hz}, 2 \mathrm{H}, \mathrm{CH}_{2} \mathrm{O}\right), 4.90(\mathrm{~d}, J 1.8 \mathrm{~Hz}, 1 \mathrm{H}$, CCHO), 5.10 (ap s, 2H, CH $2 \mathrm{Ph}$ ), 5.17 (ap.s, 2H, $\mathrm{CH}_{2} \mathrm{Ph}$ ), 5.18-5.23 (m, 1H, $\mathrm{CHCH}_{2}$ ), 7.17-7.28 (m, 2H), 7.33-7.41 (m, 8H). $\left.{ }^{13} \mathrm{C} \mathrm{NMR} \mathrm{(50} \mathrm{MHz,} \mathrm{CDCl}_{3}\right): \delta$ (ppm) 14.0, 22.7, 24.8, 25.0, 29.1, 29.3, 29.4, 29.6, 31.9, 34.0, 61.9, 67.5, 73.8, 121.6, 128.0, 128.7, 128.8, 128.9, 135.2, 136.0, 155.1, 168.5, 172.2, 173.0. MS (ESI positive) $\mathrm{m} / \mathrm{z}$ (\%): 744 $[\mathrm{M}+\mathrm{Na}]^{+},(100)$. Elemental Analysis: $\mathrm{C}_{44} \mathrm{H}_{64} \mathrm{O}_{8} \mathrm{C} 73.30 \%, \mathrm{H} 8.95 \%$. Found: $\mathrm{C} 73.46 \%, \mathrm{H} 8.92 \%$.

(S)-2-Acetoxy-2-((R)-3,4-bis(benzyloxy)-5-oxo-2,5-dihydrofuran-2-yl)ethyl dodecanoate (3d). Acetyl chloride (154 mg, $1.95 \mathrm{mmol}, 1.5$ eq.) was slowly added, to a cooled solution $\left(0^{\circ} \mathrm{C}\right)$ of $\mathbf{5 d}$ (vide infra) $(700 \mathrm{mg}, 1.30$ mmol, 1.0 eq.) and $\mathrm{Et}_{3} \mathrm{~N}$ (400 mg, $3.9 \mathrm{mmol}, 3.0$ eq.) in $\mathrm{CH}_{2} \mathrm{Cl}_{2}(10 \mathrm{~mL}$ ). The reaction was allowed to warm up to $\mathrm{rt}$ and the mixture was stirred overnight under inert atmosphere. The reaction was then diluted with $\mathrm{H}_{2} \mathrm{O}$ $(10 \mathrm{~mL})$, the layers separated and the aqueous layer extracted into $\mathrm{Et}_{2} \mathrm{O}(2 \times 10 \mathrm{~mL})$. The combined organic layers were dried over $\mathrm{Na}_{2} \mathrm{SO}_{4}$, filtered, concentrated in vacuo and purified by flash column chromatography (petroleum ether/EtOAc 4:1) to give $3 \mathbf{d}(695 \mathrm{mg}, 92 \%)$ as a colourless oil. ${ }^{1} \mathrm{H} \mathrm{NMR}(200 \mathrm{MHz}, \mathrm{CDCl} 3): \delta(\mathrm{ppm})$ 0.87 (ap t, J $7.0 \mathrm{~Hz}, 3 \mathrm{H}), 1.24(\mathrm{~m}, 16 \mathrm{H}), 1.42-1.63(\mathrm{~m}, 2 \mathrm{H}), 1.93(\mathrm{~s}, 3 \mathrm{H}), 2.27(\mathrm{t}, J 8 \mathrm{~Hz}, 2 \mathrm{H}), 4.25$ (dd, J 7.3, 12.0 $\mathrm{Hz}, 1 \mathrm{H}, \mathrm{CH}_{\mathrm{a}} \mathrm{H}_{\mathrm{b}} \mathrm{O}$ ), 4.32 (dd, J 6.6, $12.0 \mathrm{~Hz}, 1 \mathrm{H}, \mathrm{CH}_{\mathrm{a}} \mathrm{H}_{\mathrm{b}} \mathrm{O}$ ), 4.80 (d, J 2.2 Hz, 1H, CHO), 5.06-5.13 (m, $2 \mathrm{H}$ ), 5.15 (ap. $\left.\mathrm{s}, 2 \mathrm{H}, \mathrm{CH}_{2} \mathrm{Ph}\right), 5.26-5.39(\mathrm{~m}, 1 \mathrm{H}), 7.17-7.26(\mathrm{~m}, 2 \mathrm{H}), 7.33-7.40(\mathrm{~m}, 8 \mathrm{H}) .{ }^{13} \mathrm{C} \mathrm{NMR}\left(50 \mathrm{MHz}, \mathrm{CDCl}_{3}\right): \delta(\mathrm{ppm}) 14.1$, 20.4, 22.6, 24.7, 29.1, 29.2, 29.3, 29.4, 29.6, 31.9, 33.9, 61.8, 67.7, 73.7, 121.4, 128.0, 128.4, 128.8, 129.0, 135.1, 135.9, 155.1, 168.5, 169.4, 173.0. MS (ESI positive) $\mathrm{m} / \mathrm{z}(\%): 603$ [M+Na] $^{+},(100)$. Elemental Analysis: $\mathrm{C}_{34} \mathrm{H}_{44} \mathrm{O}_{8} \mathrm{C} 70.32 \%, \mathrm{H} 7.64 \%$. Found: C $70.08 \%, \mathrm{H} 7.67 \%$.

(S)-1-((R)-3,4-Bis(benzyloxy)-5-oxo-2,5-dihydrofuran-2-yl)ethane-1,2-diyl dioleate (3e). Following the general procedure GP2, 2a (500 mg, $1.40 \mathrm{mmol}$ ) and oleoyl chloride (969 $\mathrm{mg}, 3.22 \mathrm{mmol}$ ) gave, after flash chromatography, 3 e (966 mg, 78\%) as a slightly yellowish oil. ${ }^{1} \mathrm{H}$ NMR (200 MHz, CDCl 3 ): $\delta(p p m) 0.88$ (ap t, J $6.4 \mathrm{~Hz}, 6 \mathrm{H}), 1.17-1.40(\mathrm{~m}, 40 \mathrm{H}), 1.48-1.68(\mathrm{~m}, 4 \mathrm{H}), 1.93-2.08(\mathrm{~m}, 8 \mathrm{H}), 2.18-2.31(\mathrm{~m}, 4 \mathrm{H}), 4.23(\mathrm{dd}, J 7.1,11.5$ $\left.\mathrm{Hz}, 1 \mathrm{H}, \mathrm{CH}_{\mathrm{a}} \mathrm{H}_{\mathrm{b}} \mathrm{O}\right), 4.34$ (dd, J 5.0, $\left.11.5 \mathrm{~Hz}, 1 \mathrm{H}, \mathrm{CH}_{\mathrm{a}} \mathrm{H}_{\mathrm{b}} \mathrm{O}\right), 4.80$ (d, J $\left.2.1 \mathrm{~Hz}, 1 \mathrm{H}, \mathrm{CHO}\right), 5.11(\mathrm{~s}, 2 \mathrm{H}), 5.16(\mathrm{~s}, 2 \mathrm{H})$, 5.26-5.44 (m, 5H), 7.21-7.43 (m, 10H). $\left.{ }^{13} \mathrm{C} \mathrm{NMR} \mathrm{(50} \mathrm{MHz,} \mathrm{CDCl} 3\right): \delta(p p m) ~ 14.0,22.6,24.8,25.0,27.2,29.1$, 29.3, 29.5, 29.7, 29.8, 31.9, 34.0, 61.9, 67.6, 73.7, 121.7, 128.0, 128.2, 128.7, 128.8, 128.9, 129.7, 130.0, 135.2, 136.1, 155.0, 168.4, 172.1, 172.9. MS (ESI positive) $\mathrm{m} / z$ (\%): 908 [M+Na] ${ }^{+}$, (100). Elemental Analysis: $\mathrm{C}_{56} \mathrm{H}_{84} \mathrm{O}_{8} \mathrm{C}$ $75.98 \%$, H 9.56\%. Found: C 75.77\%, H 9.58\%.

(S)-1-((R)-3,4-Bis((4-methoxybenzyl)oxy)-5-oxo-2,5-dihydrofuran-2-yl)ethane-1,2-diyl dioleate (3f). Following the general procedure GP2, $\mathbf{2 b}(200 \mathrm{mg}, 0.48 \mathrm{mmol})$ and oleoyl chloride (301 $\mathrm{mg}, 1.0 \mathrm{mmol})$ gave, after flash chromatography, $3 f$ (376 mg, 83\%) as a slightly yellowish oil. ${ }^{1} \mathrm{H}$ NMR (200 MHz, $\left.\mathrm{CDCl}_{3}\right): \delta(\mathrm{ppm}) 0.83-0.91$ (m, $6 \mathrm{H}), 1.19-1.29(\mathrm{~m}, 40 \mathrm{H}), 1.41-1.66(\mathrm{~m}, 4 \mathrm{H}), 1.87-2.05(\mathrm{~m}, 8 \mathrm{H}), 2.17-2.36(\mathrm{~m}, 4 \mathrm{H}), 3.78(\mathrm{~s}, 3 \mathrm{H}), 3.79(\mathrm{~s}, 3 \mathrm{H}), 4.19$ 
(dd, J 7.3, 11.7 Hz, 1H, $\mathrm{CH}_{\mathrm{a}} \mathrm{H}_{\mathrm{b}} \mathrm{O}$ ), 4.31 (dd, J 5.5, $11.7 \mathrm{~Hz}, 1 \mathrm{H}, \mathrm{CH}_{\mathrm{a}} \mathrm{H}_{\mathrm{b}} \mathrm{O}$ ) 4.75 (d, J 2.2 Hz, 1H, CHO), 5.06 (ap. s, $4 \mathrm{H}, \mathrm{CH}_{2} \mathrm{Ar}$ ), 5.27-5.38 (m, 5H), 6.83-6.91 (m, 4H), 7.17 (ap d, Is $8.8 \mathrm{~Hz}, 2 \mathrm{H}$ ), 7.32 (ap d, Is $8.8 \mathrm{~Hz}, 2 \mathrm{H}$ ). ${ }^{13} \mathrm{C} \mathrm{NMR}$ $\left(50 \mathrm{MHz}, \mathrm{CDCl}_{3}\right.$ ): $\delta$ (ppm) 14.0, 22.6, 24.7, 24.9, 27.1, 29.0, 29.1, 29.2, 29.5, 29.6, 29.7, 31.8, 33.9, 55.2, 61.8, 67.4, 73.3, 73.4, 73.7, 114.0, 121.2, 127.2, 128.1, 129.6, 129.9, 130.6, 155.2, 160.0, 168.6, 172.1, 172.9. MS (ESI positive) $\mathrm{m} / z$ (\%): $968[\mathrm{M}+\mathrm{Na}]^{+}$, (100). Elemental Analysis: $\mathrm{C}_{58} \mathrm{H}_{88} \mathrm{O}_{10} \mathrm{C} 73.69 \%, \mathrm{H} 9.38 \%$. Found: C 73.91\%, H 9.40\%.

(S)-1-((R)-3,4-Bis(benzyloxy)-5-oxo-2,5-dihydrofuran-2-yl)ethane-1,2-diyl diacrylate (3g). Following the general procedure GP2, 2a (200 mg, $0.56 \mathrm{mmol}$ ) and acryloyl chloride (117 mg, $1.29 \mathrm{mmol})$ gave, after flash chromatography, $\mathbf{3 g}(195 \mathrm{mg}, 75 \%)$ as a colourless oil. ${ }^{1} \mathrm{H}$ NMR $\left(200 \mathrm{MHz}, \mathrm{CDCl}_{3}\right): \delta(\mathrm{ppm})$ 4.36-4.45 (m, $2 \mathrm{H}$, $\mathrm{CH}_{2} \mathrm{O}$ ), 4.87 (d, J $\left.2.2 \mathrm{~Hz}, 1 \mathrm{H}, \mathrm{CHO}\right), 5.06$ (d, J 10.2, 1H, $\mathrm{CH}_{2} \mathrm{Ph}$ ), 5.08 (ap. s, 2H, CH $\mathrm{CH}_{2} \mathrm{Ph}, 5.14$ (d, J 10.2, 1H, $\left.\mathrm{CH}_{2} \mathrm{Ph}\right), 5.42-5.56(\mathrm{~m}, 1 \mathrm{H}), 5.81-6.19(\mathrm{~m}, 4 \mathrm{H}), 6.37-6.51(\mathrm{~m}, 2 \mathrm{H}), 7.19-7.38(\mathrm{~m}, 10 \mathrm{H}) .{ }^{13} \mathrm{C} \mathrm{NMR}\left(50 \mathrm{MHz}, \mathrm{CDCl}_{3}\right)$ : $\delta$ (ppm) 62.0, 67.6, 73.6, 73.7, 73.9, 121.7, 127.9, 127.2, 127.5, 128.0, 128.6, 128.7, 128.9, 131.8, 132.5, 134.9, 135.8, 155.2, 164.3, 165.2, 168.6. MS (ESI positive) $\mathrm{m} / \mathrm{z}(\%): 487$ [M+Na] ${ }^{+}$, (100). Elemental Analysis: $\mathrm{C}_{26} \mathrm{H}_{24} \mathrm{O}_{8} \mathrm{C}$ $67.23 \%$, H 5.21\%. Found: C 67.13\%, H 5.22\%.

Cleavage of benzyl ethers. General procedure (GP3). To a solution of ascorbyl 5-O-,6-O-dialkanoate-2,3dibenzyl ethers 3a-d $(3.0 \mathrm{mmol})$ in EtOAc $(30 \mathrm{~mL}), \mathrm{Pd} / \mathrm{C}(10 \%)$ was added. Then a balloon filled with $\mathrm{H}_{2}$ was attached to the flask and the reaction was stirred for $2 \mathrm{~h}$. The reaction progress was monitored by TLC. After the complete consumption of the starting product, the mixture was filtered through Celite and the solvent was removed under reduced pressure. Crystallization from $\mathrm{Et}_{2} \mathrm{O} /$ petroleum ether gave ascorbyl 5-O-,6-Odialkanoates $4 a-d$ in almost quantitative yield.

(S)-1-((R)-3,4-Dihydroxy-5-oxo-2,5-dihydrofuran-2-yl)ethane-1,2-diyl dioctanoate (4a). Prepared from 3a

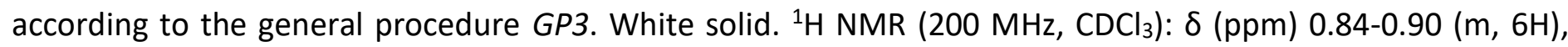
1.22-1.29 (m, 16H), 1.51-1.67 (m, 4H), 2.28-2.36 (m, 4H), $4.29\left(\mathrm{dd}, J 6.6,11.8 \mathrm{~Hz}, 1 \mathrm{H}, \mathrm{CH}_{\mathrm{a}} \mathrm{H}_{\mathrm{b}} \mathrm{O}\right), 4.42(\mathrm{dd}, J 4.8$, 11.8.0 Hz, $\left.1 \mathrm{H}, \mathrm{CH}_{\mathrm{a}} \mathrm{H}_{\mathrm{b}} \mathrm{O}\right), 4.91(\mathrm{~d}, J 3.4 \mathrm{~Hz}, 1 \mathrm{H}, \mathrm{CHO}), 5.38-5.46(1 \mathrm{H}, \mathrm{m}, \mathrm{CHO}) .{ }^{13} \mathrm{C} \mathrm{NMR}\left(100 \mathrm{MHz}^{\mathrm{C}} \mathrm{CDCl}_{3}\right): \delta$ (ppm) 13.9, 22.5, 24.7, 24.8, 28.8, 28.9, 29.0, 31.5, 34.0, 62.0, 68.2, 74.6, 119.5, 148.9, 170.1, 172.9, 173.7. Elemental Analysis: $\mathrm{C}_{22} \mathrm{H}_{36} \mathrm{O}_{8} \mathrm{C} 61.66 \%, \mathrm{H} 8.47 \%$. Found: $\mathrm{C} 61.59 \%, \mathrm{H} 8.50 \%$.

(S)-1-((R)-3,4-Dihydroxy-5-oxo-2,5-dihydrofuran-2-yl)ethane-1,2-diyl didecanoate (4b). Prepared from 3b according to the general procedure GP3. White solid. $\left.{ }^{1} \mathrm{H} \mathrm{NMR} \mathrm{(400} \mathrm{MHz,CDCl} 3\right): \delta(p p m) 0.87$ (ap t, Is $6.6 \mathrm{~Hz}$, $6 \mathrm{H}), 1.26(\mathrm{~m}, 24 \mathrm{H}), 1.51-1.68(\mathrm{~m}, 4 \mathrm{H}), 2.29-2.38(\mathrm{~m}, 4 \mathrm{H}), 4.30\left(\mathrm{dd}, J 6.8,11.6 \mathrm{~Hz}, 1 \mathrm{H}, \mathrm{CH}_{\mathrm{a}} \mathrm{H}_{\mathrm{b}} \mathrm{O}\right), 4.42(\mathrm{dd}, J 4.8$, $\left.11.6 \mathrm{~Hz}, 1 \mathrm{H}, \mathrm{CH}_{\mathrm{a}} \mathrm{H}_{\mathrm{b}} \mathrm{O}\right), 4.92(\mathrm{~d}, J 3.6 \mathrm{~Hz}, 1 \mathrm{H}, \mathrm{CHO}), 5.41-5.45\left(\mathrm{~m}, 1 \mathrm{H}, \mathrm{CHCH}_{2}\right) .{ }^{13} \mathrm{C} \mathrm{NMR}(50 \mathrm{MHz}, \mathrm{CDCl}): \delta(\mathrm{ppm})$ 13.9, 22.5, 24.6, 24.7, 28.9, 29.0, 29.1, 29.3, 31.7, 33.9, 34.0, 62.1, 68.1, 74.8, 119.1, 150.9, 171.5, $173.1,173.9$ ppm. MS (ESI negative) $\mathrm{m} / \mathrm{z}(\%): 483[\mathrm{M}-\mathrm{H}]^{-},(100) . \mathrm{IR}\left[\mathrm{CDCl}_{3}\right] \vee\left(\mathrm{cm}^{-1}\right): 3350-3400,3100-3200,2800-2950$, 1550, 1690, 1620, 1100-1200. Elemental Analysis: $\mathrm{C}_{26} \mathrm{H}_{44} \mathrm{O}_{8} \mathrm{C} 64.44 \%, \mathrm{H}$ 9.15\%. Found: C 64.09\%, $\mathrm{H} 9.25 \%$.

(S)-1-((R)-3,4-Dihydroxy-5-oxo-2,5-dihydrofuran-2-yl)ethane-1,2-diyl didodecanoate (4c). Prepared from 3c according to the general procedure $G P 3$. White solid ${ }^{1} \mathrm{H} N M R\left(400 \mathrm{MHz}, \mathrm{CDCl}_{3}\right): \delta(\mathrm{ppm}) 0.88(\mathrm{~m}, 6 \mathrm{H}), 1.26(\mathrm{~m}$, $32 \mathrm{H}), 1.54-1.65(\mathrm{~m}, 4 \mathrm{H}), 2.29-2.34(\mathrm{~m}, 4 \mathrm{H}), 4.31\left(\mathrm{dd}, J 6.6,11.7 \mathrm{~Hz}, 1 \mathrm{H}, \mathrm{CH}_{\mathrm{a}} \mathrm{H}_{\mathrm{b}} \mathrm{O}\right), 4.41(\mathrm{dd}, J 4.8,11.7 \mathrm{~Hz}, 1 \mathrm{H}$, $\left.\mathrm{CH}_{\mathrm{a}} \mathrm{H}_{\mathrm{b}} \mathrm{O}\right), 4.90$ (d, J $\left.3.1 \mathrm{~Hz}, 1 \mathrm{H}, \mathrm{CHO}\right), 5.38-5.43\left(\mathrm{~m}, 1 \mathrm{H}, \mathrm{CHCH}_{2}\right) .{ }^{13} \mathrm{C} \mathrm{NMR}\left(100 \mathrm{MHz}, \mathrm{CDCl}_{3}\right): \delta(\mathrm{ppm})$ 13.9, 22.5, 24.6, 24.7, 28.9, 29.0, 29.2, 29.4, 29.5, 31.8, 33.8, 33.9, 62.1, 68.1, 74.7, 118.8, 151.4, 171.5, 173.0, 173.8. MS (ESI negative) $\mathrm{m} / z(\%): 539[\mathrm{M}-\mathrm{H}]^{-},(100) . \mathrm{IR}\left[\mathrm{CDCl}_{3}\right] \vee\left(\mathrm{cm}^{-1}\right): 3300-3500,2800-2950,1770,1690,1100-1190$. Elemental Analysis: $\mathrm{C}_{30} \mathrm{H}_{52} \mathrm{O}_{8} \mathrm{C} 64.64 \%, \mathrm{H} 9.69 \%$. Found $\mathrm{C} 64.32 \%, \mathrm{H} 9.59 \%$.

(S)-2-Acetoxy-2-((R)-3,4-dihydroxy-5-oxo-2,5-dihydrofuran-2-yl)ethyl dodecanoate (4d). Prepared from 3d according to the general procedure GP3. White solid ${ }^{1} \mathrm{H} N M R\left(400 \mathrm{MHz}, \mathrm{CDCl}_{3}\right): \delta(\mathrm{ppm}) 0.87(\mathrm{t}, J 6.8 \mathrm{~Hz}, 3 \mathrm{H})$, 1.19-1.38 (m, 16H), 1.54-1.69 (m, 2H), $2.09(\mathrm{~s}, 3 \mathrm{H}), 2.34(\mathrm{t}, J 7.6 \mathrm{~Hz}, 2 \mathrm{H}), 4.31\left(\mathrm{dd}, J 6.6,11.8 \mathrm{~Hz}, 1 \mathrm{H}, \mathrm{CH}_{\mathrm{a}} \mathrm{H}_{\mathrm{b}} \mathrm{O}\right)$, 
4.42 (dd, J 4.8, $11.8 \mathrm{~Hz}, 1 \mathrm{H}, \mathrm{CH}_{\mathrm{a}} \mathrm{H}_{\mathrm{b}} \mathrm{O}$ ), 4.95 (d, J $\left.3.5 \mathrm{~Hz}, 1 \mathrm{H}, \mathrm{CHO}\right), 5.38-5.46(1 \mathrm{H}, \mathrm{m}, \mathrm{CHO}) .{ }^{13} \mathrm{C} \mathrm{NMR}(100 \mathrm{MHz}$, $\left.\mathrm{CDCl}_{3}\right): \delta$ (ppm) 14.1, 20.6, 22.7, 24.8, 29.1, 29.2, 29.3, 29.4, 29.6, 31.9, 34.0, 61.9, 68.5, 74.7, 119.2, 150.4, 170.4, 171.3, 173.9. MS (ESI negative) $\mathrm{m} / \mathrm{z}$ (\%): 399 [M-H] $]^{-},(100)$. Elemental Analysis: $\mathrm{C}_{20} \mathrm{H}_{32} \mathrm{O}_{8} \mathrm{C} 59.98 \%, \mathrm{H}$ 8.05\%. Found C 59.63\%, H 8.19\%.

(S)-1-((R)-3,4-Dihydroxy-5-oxo-2,5-dihydrofuran-2-yl)ethane-1,2-diyl dioleate (4e). To a solution of $\mathbf{3 f}$ (155 $\mathrm{mg}, 0.16 \mathrm{mmol}, 1$ eq.) and benzenethiol ( $43 \mathrm{mg}, 0.38 \mathrm{mmol}, 2.4$ eq.) in dry $\mathrm{CH}_{2} \mathrm{Cl}_{2}$ ( $3 \mathrm{~mL}$ ) cooled at $-78^{\circ} \mathrm{C}, \mathrm{SnCl}_{4}$ (39.4 mL, $0.336 \mathrm{mmol}, 2.1$ eq.) was dropwise added and the reaction was stirred for $30 \mathrm{~min}$. Then the mixture was quenched with saturated aqueous $\mathrm{NaHCO}_{3}$, allowed to warm up at $\mathrm{rt}$ and extracted with EtOAc, dried over $\mathrm{Na}_{2} \mathrm{SO}_{4}$, filtered and concentrated in vacuo. The residue was purified by column chromatography (petroleum ether/EtOAc $1: 1)$ to give 4 e $(38 \mathrm{mg}, 34 \%)$ as a slightly yellow sticky solid. ${ }^{1} \mathrm{H} \mathrm{NMR}(200 \mathrm{MHz}$, $\left.\mathrm{CDCl}_{3}\right): \delta(\mathrm{ppm})$ 0.83-0.95 (m, 6H), 1.21-1.32 (m, 40H), 1.46-1.63 (m, 4H), 1.93-2.04 (m, 8H), 2.24-2.36 (m, 4H), 4.27 (dd, J 6.6, $\left.11.9 \mathrm{~Hz}, 1 \mathrm{H}, \mathrm{CH}_{\mathrm{a}} \mathrm{H}_{\mathrm{b}} \mathrm{O}\right), 4.41$ (dd, J 5.0, $\left.11.9 \mathrm{~Hz}, 1 \mathrm{H}, \mathrm{CH}_{\mathrm{a}} \mathrm{H}_{\mathrm{b}} \mathrm{O}\right), 4.89(\mathrm{~d}, J 2.6 \mathrm{~Hz}, 1 \mathrm{H}, \mathrm{CHO}), 5.31-$ $5.36(\mathrm{~m}, 4 \mathrm{H}), 5.37-5.47(\mathrm{~m}, 1 \mathrm{H}) .{ }^{13} \mathrm{C} \mathrm{NMR}\left(50 \mathrm{MHz}, \mathrm{CDCl}_{3}\right): \delta$ (ppm) 13.8, 22.6, 24.8, 24.8, 27.0, 29.0, 29.1, 29.3, 29.4, 29.6, 29.7, 31.9, 34.2, 61.8, 67.4, 73.5, 129.7, 130.0, 155.3, 168.9, 172.6, 173.8. MS (ESI negative) $\mathrm{m} / \mathrm{z}$ (\%): 704 [M-H]', (100). Elemental Analysis: $\mathrm{C}_{42} \mathrm{H}_{72} \mathrm{O}_{8} \mathrm{C} 71.55 \%, \mathrm{H} 10.29 \%$. Found: $\mathrm{C} 71.38 \%, \mathrm{H} 10.31 \%$.

(S)-2-((R)-3,4-Bis((4-methoxybenzyl)oxy)-5-oxo-2,5-dihydrofuran-2-yl)-2-hydroxyethyl oleate (5a). Oleoyl chloride (331 mg, $1.1 \mathrm{mmol}$ ) was slowly added to a solution of $\mathbf{2 b}\left(416 \mathrm{mg}, 1.0 \mathrm{mmol}\right.$ ) and Et $\mathrm{H}_{3} \mathrm{~N}(507 \mathrm{mg}, 5.0$ $\mathrm{mmol})$ in $\mathrm{CH}_{2} \mathrm{Cl}_{2}(10 \mathrm{~mL})$. The reaction was stirred overnight under inert atmosphere at ambient temperature. Afterwards, the mixture was diluted with $\mathrm{H}_{2} \mathrm{O}(10 \mathrm{~mL})$ and the aqueous layer extracted into $\mathrm{Et}_{2} \mathrm{O}(2 \times 10 \mathrm{~mL})$. The combined organic layers were dried over $\mathrm{Na}_{2} \mathrm{SO}_{4}$, filtered, concentrated in vacuo and purified by flash column chromatography (petroleum ether/EtOAc 4:1) to give the product 5a (327 mg, 48\%). Colourless oil ${ }^{1} \mathrm{H}$ NMR (200 MHz, $\left.\mathrm{CDCl}_{3}\right): \delta(\mathrm{ppm})$ 0.83-0.91 (m, 3H), 1.19-1.29 (m, 20H), 1.47-1.61 (m, 2H), 1.87-2.11 (m, 4H), 2.17-2.35 (m, 2H), $3.79(\mathrm{~s}, 3 \mathrm{H}), 3.81(\mathrm{~s}, 3 \mathrm{H}), 4.02-4.36(\mathrm{~m}, 3 \mathrm{H}), 4.62(\mathrm{~d}, J 2.2 \mathrm{~Hz}, 1 \mathrm{H}, \mathrm{CHO}), 5.07$ (ap. s, 4H), 5.29$5.36(\mathrm{~m}, 2 \mathrm{H}), 6.84-6.91(\mathrm{~m}, 4 \mathrm{H}), 7.17$ (ap d, Is $8.8 \mathrm{~Hz}, 2 \mathrm{H}$ ), 7.32 (ap d, Is $8.8 \mathrm{~Hz}, 2 \mathrm{H}$ ). MS (ESI positive) $\mathrm{m} / z(\%)$ : $704[\mathrm{M}+\mathrm{Na}]^{+}$, (100). Elemental Analysis: $\mathrm{C}_{40} \mathrm{H}_{56} \mathrm{O}{ }_{9} \mathrm{C} 70.56 \%, \mathrm{H} 8.29 \%$. Found: C $70.69 \%, \mathrm{H} 8.30 \%$.

(S)-2-((R)-3,4-Bis(benzyloxy)-5-oxo-2,5-dihydrofuran-2-yl)-2-hydroxyethyl acrylate (5b). Acryloyl chloride $(99.6 \mathrm{mg}, 1.1 \mathrm{mmol})$ was slowly added to a solution of $2 \mathrm{a}(356 \mathrm{mg}, 1.0 \mathrm{mmol})$ and $\mathrm{Et}{ }_{3} \mathrm{~N}(152 \mathrm{mg}, 1.5 \mathrm{mmol}) \mathrm{in}$ $\mathrm{CH}_{2} \mathrm{Cl}_{2}(10 \mathrm{~mL})$. The reaction was stirred overnight under inert atmosphere at ambient temperature. Afterwards, the mixture was diluted with $\mathrm{H}_{2} \mathrm{O}(10 \mathrm{~mL})$, the layers separated and the aqueous layer extracted into $\mathrm{Et}_{2} \mathrm{O}(2 \times 10 \mathrm{~mL})$. The combined organic layers were dried over $\mathrm{Na}_{2} \mathrm{SO}_{4}$, filtered, concentrated in vacuo and purified by flash column chromatography (petroleum ether/EtOAc 3:1) to give the product $5 \mathbf{b}$ (218 mg, $53 \%)$ as a colourless oil. ${ }^{1} \mathrm{H}$ NMR $\left(200 \mathrm{MHz}_{\mathrm{CDCl}}\right): \delta(\mathrm{ppm}) 2.10(\mathrm{bs}, 1 \mathrm{H}), 3.70-4.18\left(\mathrm{~m}, 1 \mathrm{H}, \mathrm{CHCH}_{2}\right), 4.30(\mathrm{dd}, J$ 5.1, $11.7 \mathrm{~Hz}, 1 \mathrm{H}, \mathrm{CH}_{\mathrm{a}} \mathrm{H}_{\mathrm{b}} \mathrm{O}$ ), 4.40 (dd, J 6.6, $11.7 \mathrm{~Hz}, 1 \mathrm{H}, \mathrm{CH}_{\mathrm{a}} \mathrm{H}_{\mathrm{b}} \mathrm{O}$ ), 4.69 (d, J $\left.2.2 \mathrm{~Hz}, 1 \mathrm{H}, \mathrm{CHO}\right), 5.10$ (ap. s, 2H, $\left.\mathrm{CH}_{2} \mathrm{Ph}\right), 5.13\left(\mathrm{~d}, J\right.$ 10.6, $\left.1 \mathrm{H}, \mathrm{CH}_{2} \mathrm{Ph}\right), 5.22\left(\mathrm{~d}, J\right.$ 10.6, $\left.1 \mathrm{H}, \mathrm{CH}_{2} \mathrm{Ph}\right), 5.87(\mathrm{dd}, 1 \mathrm{H}, J$ 1.5, $10.3 \mathrm{~Hz}), 6.13(\mathrm{dd}, 1 \mathrm{H}, J 10.3$, $\left.17.2 \mathrm{~Hz}), 6.45(\mathrm{dd}, 1 \mathrm{H}, J 1.8,17.2 \mathrm{~Hz}), 7.19-7.26(\mathrm{~m}, 4 \mathrm{H}), 7.33-7.41(\mathrm{~m}, 6 \mathrm{H}) .{ }^{13} \mathrm{C} \mathrm{NMR}(50 \mathrm{MHz}, \mathrm{CDCl})_{3}\right): \delta(\mathrm{ppm})$ $64.9,67.9,73.6,73.9,75.4,121.7,127.9,128.7,128.8,129.1,131.8,135.2,135.9,156.5,165.9,169.3 . M S$ (ESI positive) $\mathrm{m} / \mathrm{z}$ (\%): $433[\mathrm{M}+\mathrm{Na}]^{+},(100)$. Elemental Analysis: $\mathrm{C}_{23} \mathrm{H}_{22} \mathrm{O}_{7} \mathrm{C} 67.31 \%, \mathrm{H} 5.40 \%$. Found: $\mathrm{C} 67.21 \%, \mathrm{H}$ $5.41 \%$.

(S)-2-((R)-3,4-Bis((4-methoxybenzyl)oxy)-5-oxo-2,5-dihydrofuran-2-yl)-2-hydroxyethyl acrylate (5c). Following the same procedure as described above for $\mathbf{5 b}$, acryloyl chloride (72 $\mathrm{mg}, 0.79 \mathrm{mmol}$ ) and $\mathbf{2 b}$ (300 $\mathrm{mg}, 0.72 \mathrm{mmol}$ ) gave, after purification by column chromatography (petroleum ether/EtOAc 2:1), 5c (162 mg, 48\%). Colourless oil. ${ }^{1} \mathrm{H}$ NMR (200 MHz, $\left.\mathrm{CDCl}_{3}\right): \delta$ (ppm) 2.20 (bs, $\left.1 \mathrm{H}\right), 3.79(\mathrm{~s}, 3 \mathrm{H}), 3.81(\mathrm{~s}, 3 \mathrm{H}), 4.03-4.12(\mathrm{~m}$, $1 \mathrm{H}, \mathrm{CHCH}_{2}$ ), 4.22 (dd, J 5.4, $11.4 \mathrm{~Hz}, 1 \mathrm{H}, \mathrm{CH}_{\mathrm{a}} \mathrm{H}_{\mathrm{b}} \mathrm{O}$ ), 4.37 (dd, J 6.8, $\left.11.4 \mathrm{~Hz}, 1 \mathrm{H}, \mathrm{CH}_{\mathrm{a}} \mathrm{H}_{\mathrm{b}} \mathrm{O}\right), 4.65(\mathrm{~d}, J 2.2 \mathrm{~Hz}, 1 \mathrm{H}$, 
CHO), 5.02 (d, J 12.2, 1H), 5.05 (ap. s, 2H), 5.12 (d, J 12.2, 1H), 5.85 (dd, 1H, J 1.8, $10.6 \mathrm{~Hz}$ ), 6.11 (dd, 1H, J 10.4, $17.2 \mathrm{~Hz}$ ), 6.42 (dd, $1 \mathrm{H}, J$ 1.8, $17.2 \mathrm{~Hz}$ ), 6.82-6.91 (m, 4H), 7.14 (ap d, Is 7.14 Hz, 2H), 7.32 (ap d, Is 7.14 Hz, 2H). Elemental Analysis: $\mathrm{C}_{25} \mathrm{H}_{26} \mathrm{O}_{9} \mathrm{C} 63.82 \%, \mathrm{H} 5.57 \%$. Found: $\mathrm{C} 63.73 \%, \mathrm{H} 5.58 \%$.

(S)-2-((R)-3,4-Bis(benzyloxy)-5-oxo-2,5-dihydrofuran-2-yl)-2-hydroxyethyl dodecanoate (5d). Following the same procedure as described for $5 \mathrm{a}$, dodecanoyl chloride $(0.90 \mathrm{~g}, 4.14 \mathrm{mmol}), 2 \mathrm{a}(1.34 \mathrm{~g}, 3.76 \mathrm{mmol})$ and $\mathrm{Et}_{3} \mathrm{~N}$ (1.90 g, $18.8 \mathrm{mmol})$ gave, after purification by column chromatography (petroleum ether/EtOAc 3:1), 5d (1.38 $\mathrm{g}, 68 \%)$ as a colourless oil. ${ }^{1} \mathrm{H}$ NMR $\left(200 \mathrm{MHz}, \mathrm{CDCl}_{3}\right): \delta(\mathrm{ppm}) 0.88(\mathrm{ap} \mathrm{t}, J 7.2 \mathrm{~Hz}, 3 \mathrm{H}), 1.25(\mathrm{~m}, 16 \mathrm{H}), 1.48-1.61$ $(\mathrm{m}, 2 \mathrm{H}), 2.33(\mathrm{t}, J 8 \mathrm{~Hz}, 2 \mathrm{H}), 4.01-4.11(\mathrm{~m}, 1 \mathrm{H}), 4.21\left(\mathrm{dd}, J 5.0,11.9 \mathrm{~Hz}, 1 \mathrm{H}, \mathrm{CH}_{\mathrm{a}} \mathrm{H}_{\mathrm{b}} \mathrm{O}\right), 4.36(\mathrm{dd}, J 6.2,11.9 \mathrm{~Hz}, 1 \mathrm{H}$, $\mathrm{CH}_{\mathrm{a}} \mathrm{H}_{\mathrm{b}} \mathrm{O}$ ), 4.67 (d, J $\left.1.8 \mathrm{~Hz}, 1 \mathrm{H}, \mathrm{CHO}\right), 5.11$ (ap. s, 2H, $\left.\mathrm{CH}_{2} \mathrm{Ph}\right), 5.11$ (d, J 11.9, 1H, CH $2 \mathrm{Ph}$ ), 5.22 (d, J 11.9, 1H, $\left.\mathrm{CH}_{2} \mathrm{Ph}\right), 7.18-7.26(\mathrm{~m}, 2 \mathrm{H}), 7.33-7.41(\mathrm{~m}, 8 \mathrm{H}) .{ }^{13} \mathrm{C} \mathrm{NMR}\left(50 \mathrm{MHz}, \mathrm{CDCl}_{3}\right): \delta(\mathrm{ppm}) 14.0,22.5,24.8,29.0,29.1$, 29.3, 29.4, 29.5, 31.6, 34.0, 64.1, 67.6, 73.1, 73.8, 75.7, 121.3, 127.8, 128.2, 128.4, 128.7, 135.1, 135.6, 156.8, 169.7, 173.5. Elemental Analysis: $\mathrm{C}_{32} \mathrm{H}_{42} \mathrm{O}_{7} \mathrm{C} 71.35 \%, \mathrm{H} 7.86 \%$. Found: $\mathrm{C} 71.13 \%, \mathrm{H} 7.93 \%$.

(S)-1-((R)-3,4-Bis(benzyloxy)-5-oxo-2,5-dihydrofuran-2-yl)-2-(trityloxy)-ethyl-dodecanoate (7a). Dodecanoyl chloride (279 mg, $1.28 \mathrm{mmol})$ was slowly added to a solution of $(R)-3,4-$ bis(benzyloxy)-5-((S)-1-hydroxy-2(trityloxy)ethyl)furan-2(5H)-one $6 \mathrm{a}^{24}(700 \mathrm{mg}, 1.16 \mathrm{mmol}), 4-D M A P(212 \mathrm{mg}, 1.74 \mathrm{mmol})$ and DCC (358 mg, $1.74 \mathrm{mmol})$ in $\mathrm{MeCN}(13 \mathrm{~mL})$. The reaction mixture was stirred for $18 \mathrm{~h}$ at $\mathrm{rt}$ under an inert atmosphere. The solvent was removed under reduced pressure and the crude material was purified by column chromatography (petroleum ether/EtOAc 8:1) to give $7 a(842 \mathrm{mg}, 93 \%)$. Colourless oil. ${ }^{1} \mathrm{H}$ NMR $\left(200 \mathrm{MHz}, \mathrm{CDCl}_{3}\right) \delta(\mathrm{ppm}): 0.88$ (ap t, Is $6.5 \mathrm{~Hz}, 3 \mathrm{H}$ ), 1.20-1.25 (m, 16H), 1.43-1.66 (m, 2H), 2.16-2.23 (m, 2H), 3.31 (dd, J 7.0, 9.5 Hz, $1 \mathrm{H}), 3.44$ $(\mathrm{dd}, J$ 7.0, $9.5 \mathrm{~Hz}, 1 \mathrm{H}), 4.90(\mathrm{~d}, J 1.8 \mathrm{~Hz}, 1 \mathrm{H}), 5.07(\mathrm{~s}, 2 \mathrm{H}), 5.14(\mathrm{~s}, 2 \mathrm{H}), 5.23-5.31(\mathrm{~m}, 1 \mathrm{H}), 7.19-7.43(\mathrm{~m}, 25 \mathrm{H}) .{ }^{13} \mathrm{C}$ NMR $\left(50 \mathrm{MHz}, \mathrm{CDCl}_{3}\right) \delta$ (ppm): 14.0, 22.6, 24.9, 29.1, 29.2, 29.3, 29.5, 31.8, 34.0, 61.9, 68.6, 73.7, 73.9, 87.3, 121.5, 127.1, 127.8, 128.0, 128.6, 128.7, 135.3, 136.1, 143.6, 155.5, 168.7, 172.1. MS (ESI positive) $\mathrm{m} / \mathrm{z}$ (\%): $433[\mathrm{M}+\mathrm{Na}]^{+}$, (100). Elemental Analysis: $\mathrm{C}_{51} \mathrm{H}_{56} \mathrm{O}_{7} \mathrm{C} 78.43 \%, \mathrm{H} 7.23 \%$. Found: $\mathrm{C} 78.68 \%, \mathrm{H} 7.20 \%$.

(S)-1-((R)-3,4-bis((4-methoxybenzyl)oxy)-5-oxo-2,5-dihydrofuran-2-yl)-2-(trityloxy)ethyl oleate (7b). Following the same procedure for the synthesis of 7a, oleoyl chloride (166 mg, $0.55 \mathrm{mmol}$ ) and 6b (329 mg, 0.50 ) gave, after purification by column chromatography (petroleum ether/EtOAc 8:1), product $7 \mathbf{b}$ (402 mg, 87\%). Colourless oil. ${ }^{1} \mathrm{H} N M R\left(400 \mathrm{MHz}, \mathrm{CDCl}_{3}\right) \delta(\mathrm{ppm}): 0.82(\mathrm{t}, J 6.8 \mathrm{~Hz}, 3 \mathrm{H}), 1.15-1.25(\mathrm{~m}, 20 \mathrm{H}), 1.41-1.56(\mathrm{~m}$, $2 \mathrm{H}), 1.84-1.95(\mathrm{~m}, 4 \mathrm{H}), 2.10-2.18(\mathrm{~m}, 2 \mathrm{H}), 3.25(\mathrm{dd}, J 6.8,10.0 \mathrm{~Hz}, 1 \mathrm{H}), 3.38(\mathrm{dd}, J 6.4,10.0 \mathrm{~Hz}, 1 \mathrm{H}), 3.79(\mathrm{~s}, 3 \mathrm{H})$, $3.81(\mathrm{~s}, 3 \mathrm{H}), 4.84(\mathrm{~d}, J 2.0 \mathrm{~Hz}, 1 \mathrm{H}), 5.02(\mathrm{~s}, 2 \mathrm{H}), 5.06-5.10(\mathrm{~m}, 2 \mathrm{H}), 5.18-5.22(\mathrm{~m}, 1 \mathrm{H}), 5.23-5.31(\mathrm{~m}, 2 \mathrm{H}), 6.87(\mathrm{ap}$ d, Is $8.8 \mathrm{~Hz}, 2 \mathrm{H}$ ), 6.89 (ap d, Is $8.8 \mathrm{~Hz}, 2 \mathrm{H}), 7.20-7.44$ (m, 19H). ${ }^{13} \mathrm{C} \mathrm{NMR}\left(50 \mathrm{MHz}, \mathrm{CDCl}_{3}\right)$ 14.0, 22.6, 25.0, 27.2, 29.1, 29.3, 29.5, 29.7, 29.8, 30.8, 31.9, 34.0, 34.1, 55.6, 61.4, 68.7, 70.6, 73.7, 73.8, 87.3, 113.8, 114.0, 121.6, $127.1,127.4,127.8,128.2,128.7,130.0,130.9,160.0,143.7,129.7,130.0,155.6,172.2,172.8$. Elemental Analysis: $\mathrm{C}_{59} \mathrm{H}_{70} \mathrm{O}_{9} \mathrm{C} 76.76 \%, \mathrm{H} 7.64 \%$. Found: C 76.59\%, H 7.67\%.

(S)-1-((R)-3,4-bis(benzyloxy)-5-oxo-2,5-dihydrofuran-2-yl)-2-hydroxyethyl dodecanoate (8). A solution of 7a (156 mg, $0.2 \mathrm{mmol}, 1$ eq.) in dry $\mathrm{CH}_{2} \mathrm{Cl}_{2}(2 \mathrm{~mL})$ was cooled at $0{ }^{\circ} \mathrm{C}$, then $\mathrm{BF}_{3} \cdot \mathrm{Et}_{2} \mathrm{O}(115 \mathrm{~mL}, 1.0 \mathrm{mmol}, 5.0$ eq.) was slowly added and the solution was stirred under an inert atmosphere for $1 \mathrm{~h}$. Afterwards, a saturated aqueous solution of $\mathrm{NaHCO}_{3}(3 \mathrm{~mL})$ was added and the mixture was extracted with $\mathrm{CHCl}_{3}(3 \times 5 \mathrm{~mL})$. The organic layers were dried on $\mathrm{Na}_{2} \mathrm{SO}_{4}$, filtered and the solvent was eliminated under reduced pressure. The crude material was purified by column chromatography (petroleum ether/EtOAc 8:1) affording 8 (79 mg, 73\%) as a colourless oil. ${ }^{1} \mathrm{H}$ NMR $\left(200 \mathrm{MHz}^{\mathrm{C} C D C l}{ }_{3}\right) \delta(\mathrm{ppm}): 0.89(\mathrm{ap} \mathrm{t}, J 6.3 \mathrm{~Hz}, 3 \mathrm{H}), 1.20-1.29(\mathrm{~m}, 16 \mathrm{H}), 1.45-1.65$ (m, 2H), 2.15-2.28 (m, 2H), 2.95 (bs, $1 \mathrm{H}, \mathrm{OH}), 3.80$ (ap d, Is $6.5 \mathrm{~Hz}, 2 \mathrm{H}, \mathrm{CH}_{2} \mathrm{O}$ ), $4.91(\mathrm{~d}, J 1.8 \mathrm{~Hz}, 1 \mathrm{H}), 5.09(\mathrm{~s}, 2 \mathrm{H})$, $5.18(\mathrm{~s}, 2 \mathrm{H}), 5.14-5.24(\mathrm{~m}, 1 \mathrm{H}), 7.21-7.29(\mathrm{~m}, 2 \mathrm{H}), 7.31-7.41(\mathrm{~m}, 8 \mathrm{H}) .{ }^{13} \mathrm{C} \mathrm{NMR}\left(50 \mathrm{MHz}, \mathrm{CDCl}_{3}\right) \delta(\mathrm{ppm}): 13.9$, 
22.5, 24.8, 29.0, 29.1, 29.2, 29.3, 29.4, 31.8, 33.9, 60.7, 70.1, 73.5, 73.7, 121.3, 127.1, 127.8, 128.5, 128.7, 135.2, 136.0, 155.8, 168.9, 172.7. Elemental Analysis: $\mathrm{C}_{32} \mathrm{H}_{42} \mathrm{O}_{7} \mathrm{C} 71.35 \%, \mathrm{H} 7.86 \%$. Found: C 71.13\%, $\mathrm{H} 7.89 \%$.

(S)-1-((R)-3,4-dihydroxy-5-oxo-2,5-dihydrofuran-2-yl)-2-(trityloxy)ethyl dodecanoate (9). Prepared according to the general procedure GP3 starting from 7a $(250 \mathrm{mg}, 0.32 \mathrm{mmol})$. Purification by column chromatography $\left(\mathrm{CH}_{2} \mathrm{Cl}_{2} / \mathrm{MeOH}\right.$ 10:1) gave the product 9 (168 mg, 52\%). $\left.{ }^{1} \mathrm{H} \mathrm{NMR} \mathrm{(200} \mathrm{MHz,} \mathrm{CDCl} 3\right) \delta(p p m): 0.88(a p t, J 6.2 \mathrm{~Hz}$, $3 \mathrm{H}), 1.17-1.34(\mathrm{~m}, 16 \mathrm{H}), 1.50-1.66(\mathrm{~m}, 2 \mathrm{H}), 2.21-2.28(\mathrm{~m}, 2 \mathrm{H}), 3.34(\mathrm{dd}, J 5.9,9.4 \mathrm{~Hz}), 3.46(\mathrm{dd}, J 6.6,9.4 \mathrm{~Hz})$, $4.91(\mathrm{~d}, J 2.7 \mathrm{~Hz}, 1 \mathrm{H}), 5.39-5.45(\mathrm{~m}, 1 \mathrm{H}), 7.20-7.44(\mathrm{~m}, 15 \mathrm{H}) .{ }^{13} \mathrm{C} \mathrm{NMR}\left(50 \mathrm{MHz}, \mathrm{CDCl}_{3}\right) \delta(\mathrm{ppm}): 14.0,22.5,24.5$, 24.9, 25.4, 29.1, 29.3, 29.4, 31.7, 33.9, 34.8, 61.8, 68.5, 73.5, 87.2, 121.3, 127.0, 127.8, 127.9, 128.5, 128.7, 135.2, 136.0, 143.4, 155.4, 168.2, 172.0. MS (ESI negative) $\mathrm{m} / \mathrm{z}$ (\%): 599 [M-H]', (100). Elemental Analysis: $\mathrm{C}_{37} \mathrm{H}_{44} \mathrm{O}_{7} \mathrm{C} 73.98 \%, \mathrm{H} 7.38 \%$. Found: C $73.72 \%, \mathrm{H} 7.41 \%$.

General procedure for the thiol-Michael addition on ascorbyl acrylates (GP4). Neutral alumina was added to a solution of thiol (benzenethiol or 1,2-ethanedithiol) and ascorbyl acrylate $\mathbf{3 b} \mathbf{b} \mathbf{5} \mathbf{b}$ or $\mathbf{5 c}$ in dry toluene. ${ }^{39}$ The reaction was stirred under inert atmosphere at $\mathrm{rt}$ for $4 \mathrm{~h}$. Afterwards the mixture was diluted with EtOAc, washed with $\mathrm{H}_{2} \mathrm{O}$, dried over $\mathrm{Na}_{2} \mathrm{SO}_{4}$, filtered and concentrated under reduced pressure.

(S)-2-((R)-3,4-bis(benzyloxy)-5-oxo-2,5-dihydrofuran-2-yl)-2-hydroxyethyl 3-(phenyl-thio)propanoate (11a). Following the general procedure GP4, 5b $(205 \mathrm{mg}, 0.50 \mathrm{mmol}, 1$ eq.), neutral alumina (100 $\mathrm{mg})$ and benzenethiol (66 mg, $0.60 \mathrm{mmol}, 1$ eq.) in dry toluene $(5.0 \mathrm{~mL})$, gave after flash chromatography (petroleum ether/EtOAc 3:1), 11a (239 mg, 92\%). Yellowish oil. ${ }^{1} \mathrm{H}$ NMR (400 MHz, CDCl 3 ): $\delta$ (ppm) 2.23 (bs, $1 \mathrm{H}$ ), 2.65 (ap t, J $7.4 \mathrm{~Hz}, 2 \mathrm{H}$ ), 3.16 (ap t, J 7.4 Hz, 2H), 4.02-4.11 (m, 1H, $\mathrm{CHCH}_{2}$ ), 4.22 (dd, J 5.1, $\left.11.4 \mathrm{~Hz}, 1 \mathrm{H}, \mathrm{CH}_{\mathrm{a}} \mathrm{H}_{\mathrm{b}} \mathrm{O}\right), 4.34$ (dd, J 6.6, $11.4 \mathrm{~Hz}, 1 \mathrm{H}, \mathrm{CH}_{\mathrm{a}} \mathrm{H}_{\mathrm{b}} \mathrm{O}$ ), 4.67 (d, J $2.2 \mathrm{~Hz}, 1 \mathrm{H}, \mathrm{CHO}$ ), 5.10 (ap. s, $2 \mathrm{H}, \mathrm{CH}_{2} \mathrm{Ph}$ ), 5.12 (d, J 11.2, 1H, $\mathrm{CH}_{2} \mathrm{Ph}$ ), 5.21 (d, J $\left.11.2 \mathrm{~Hz}, 1 \mathrm{H}, \mathrm{CH}_{2} \mathrm{Ph}\right), 7.20-7.38(\mathrm{~m}, 15 \mathrm{H}) .{ }^{13} \mathrm{C} \mathrm{NMR}\left(100 \mathrm{MHz}, \mathrm{CDCl}_{3}\right): \delta$ (ppm) 28.9, 34.1, 64.9, 67.6, 73.6, 73.8, 75.4, 121.1, 126.6, 127.8, 128.5, 128.6, 128.7, 129.0, 130.1, 134.8, 135.1, 135.8, 156.7, 169.4, 171.2. Elemental Analysis: $\mathrm{C}_{29} \mathrm{H}_{28} \mathrm{O}_{7} \mathrm{~S} \mathrm{C} 66.91 \%, \mathrm{H} 5.42 \%$. Found: C 66.69\%, H 5.43\%.

(S)-2-((R)-3,4-bis((4-methoxybenzyl)oxy)-5-oxo-2,5-dihydrofuran-2-yl)-2-hydroxyethyl

3-(phenylthio)propanoate (11b). Following the general procedure GP4, 5c (118 mg, $0.25 \mathrm{mmol}$, 1 eq.), neutral alumina (50 $\mathrm{mg}$ ) and benzenethiol (33 $\mathrm{mg}, 0.30 \mathrm{mmol}, 1 \mathrm{eq}$.) in dry toluene $(2.5 \mathrm{~mL})$, gave after flash chromatography (petroleum ether/EtOAc 3:1), $\mathbf{1 1 b}(131 \mathrm{mg}, 91 \%)$ as a yellowish oil. ${ }^{1} \mathrm{H} \mathrm{NMR}\left(400 \mathrm{MHz}, \mathrm{CDCl}_{3}\right): \delta(\mathrm{ppm}) 2.10$ (bs, 1H), 2.64 (ap t, J $7.3 \mathrm{~Hz}, 2 \mathrm{H}$ ), 3.14 (ap t, J $7.3 \mathrm{~Hz}, 2 \mathrm{H}), 3.79(\mathrm{~s}, 3 \mathrm{H}), 3.80(\mathrm{~s}, 3 \mathrm{H}), 3.97-4.14\left(\mathrm{~m}, 1 \mathrm{H}, \mathrm{CHCH}_{2}\right)$, 4.19 (dd, J 5.1, $11.4 \mathrm{~Hz}, 1 \mathrm{H}, \mathrm{CH}_{\mathrm{a}} \mathrm{H}_{\mathrm{b}} \mathrm{O}$ ), 4.31 (dd, J 6.7, $11.4 \mathrm{~Hz}, 1 \mathrm{H}, \mathrm{CH}_{\mathrm{a}} \mathrm{H}_{\mathrm{b}} \mathrm{O}$ ), 4.62 (d, J $\left.2.2 \mathrm{~Hz}, 1 \mathrm{H}, \mathrm{CHO}\right), 5.02$ (d, J $11.2 \mathrm{~Hz}, 1 \mathrm{H}), 5.06$ (ap. s, 2H), 5.12 (d, J $11.2 \mathrm{~Hz}, 1 \mathrm{H}), 6.83-6.92(\mathrm{~m}, 4 \mathrm{H}), 7.12-7.38$ (m, 9H). Elemental Analysis: $\mathrm{C}_{31} \mathrm{H}_{32} \mathrm{O}_{9} \mathrm{~S} \mathrm{C} 64.13 \%$, H 5.56\%. Found C 64.26\%, H 5.21\%.

(S)-1-((R)-3,4-bis(benzyloxy)-5-oxo-2,5-dihydrofuran-2-yl)ethane-1,2-diyl bis(3-(phenyl-thio)propanoate) (12). Following the general procedure GP4, 3g (116 mg, $0.25 \mathrm{mmol}, 1$ eq.), neutral alumina (50 mg) and benzenethiol (66 mg, $0.60 \mathrm{mmol}, 2.4$ eq.) in dry toluene $(2.5 \mathrm{~mL}$ ), gave after flash chromatography (petroleum ether/EtOAc 5:1), 12 (152 mg, 88\%) as a yellowish oil. $\left.{ }^{1} \mathrm{H} \mathrm{NMR} \mathrm{(400} \mathrm{MHz,} \mathrm{CDCl}_{3}\right): \delta(\mathrm{ppm})$ 2.44-2.57 (m, 2H), 2.62 (ap t, J 7.3 Hz, 2H), 3.06 (ap t, J 7.4 Hz, 2H), 3.14 (ap t, J 7.4 Hz, 2H), 4.27 (dd, J 7.2, 11.6 Hz, 1H, CHa $\mathrm{H}_{\mathrm{b}} \mathrm{O}$ ), 4.36 (dd, J 5.3, 11.6 Hz, $1 \mathrm{H}, \mathrm{CH}_{\mathrm{a}} \mathrm{H}_{\mathrm{b}} \mathrm{O}$ ), 4.80 (d, J $2.2 \mathrm{~Hz}, 1 \mathrm{H}, \mathrm{CHO}$ ), 5.12 (AB system, Is $10.8 \mathrm{~Hz}, 2 \mathrm{H}, \mathrm{CH}_{2} \mathrm{Ph}$ ), 5.20 ( $\mathrm{AB}$ system, Is $11.3 \mathrm{~Hz}, 2 \mathrm{H}, \mathrm{CH}_{2} \mathrm{Ph}$ ) 5.41 (ddd, J 2.2, 5.3, 7.2 Hz, 1H, $\mathrm{CHCH}_{2}$ ), 7.17-7.37 (m, 20H). ${ }^{13} \mathrm{C} \mathrm{NMR} \mathrm{(100}$ $\left.\mathrm{MHz}_{1} \mathrm{CDCl}_{3}\right): \delta$ (ppm) 28.8, 29.1, 33.8, 34.1, 62.2, 67.7, 73.6, 73.5, 73.6, 73.7, 121.3, 126.6, 126.7, 127.4, 128.1, 128.6, 128.7, 128.8, 128.9, 129.0, 129.1, 130.1, 130.3, 134.9, 135.0, 135.8, 154.8, 168.4, 170.2, 170.9. MS (ESI positive) $m / z$ (\%): $708[\mathrm{M}+\mathrm{Na}]^{+}$, (100). Elemental Analysis: $\mathrm{C}_{38} \mathrm{H}_{36} \mathrm{O}_{8} \mathrm{~S}_{2} \mathrm{C} 66.65 \%, \mathrm{H} 5.30 \%$. Found: $\mathrm{C} 66.78 \%, \mathrm{H}$ $5.27 \%$. 
$\operatorname{Bis((S)-2-((R)-3,4-bis(benzyloxy)-5-oxo-2,5-dihydrofuran-2-yl)-2-hydroxyethyl)~}$

3,3'-(ethane-1,2-diylbis(sulfanediyl))dipropionate (13). Following the general procedure GP4, 5b (103 mg, $0.25 \mathrm{mmol}, 1$ eq.), neutral alumina (25 mg) and 1,2-ethanedithiol (10.6 mg, $0.11 \mathrm{mmol}, 0.45 \mathrm{eq}$.) in dry toluene ( $2 \mathrm{~mL}$ ), gave after flash chromatography (petroleum ether/EtOAc 3:2), 13 ( $95 \mathrm{mg}, 94 \%)$. Slightly yellow oil. $\left.{ }^{1} \mathrm{H} \mathrm{NMR} \mathrm{(200} \mathrm{MHz,} \mathrm{CDCl}_{3}\right)$ : $\delta(\mathrm{ppm})$ 2.61-2.93 (m, 12H), $3.38(\mathrm{bd}, J 7.9 \mathrm{~Hz}, 2 \mathrm{H}, \mathrm{OH}), 4.05-4.20(\mathrm{~m}, 2 \mathrm{H}), 4.27\left(\mathrm{dd}, J\right.$ 5.6, $\left.11.3 \mathrm{~Hz}, 2 \mathrm{H}, \mathrm{CH}_{\mathrm{a}} \mathrm{H}_{\mathrm{b}} \mathrm{O}\right)$, 4.36 (dd, J 7.0, $11.3 \mathrm{~Hz}, 2 \mathrm{H}, \mathrm{CH}_{\mathrm{a}} \mathrm{H}_{\mathrm{b}} \mathrm{O}$ ), $4.74(\mathrm{~d}, J 1.9 \mathrm{~Hz}, 2 \mathrm{H}, \mathrm{CHO}), 5.06$ (ap s, 4H, CH $\left.\mathrm{CH}_{2} \mathrm{Ph}\right), 5.07(\mathrm{~d}, J 11.6 \mathrm{~Hz}, 2 \mathrm{H}$, $\left.\mathrm{CH}_{2} \mathrm{Ph}\right), 5.22\left(\mathrm{~d}, J 11.6 \mathrm{~Hz}, 4 \mathrm{H}, \mathrm{CH}_{2} \mathrm{Ph}\right), 7.17-7.26(\mathrm{~m}, 4 \mathrm{H}), 7.32-7.43(\mathrm{~m}, 16 \mathrm{H}) .{ }^{13} \mathrm{C} \mathrm{NMR}\left(50 \mathrm{MHz}, \mathrm{CDCl}_{3}\right): \delta$ (ppm) 26.6, 31.9, 35.0, 65.1, 67.7, 73.7, 74.0, 75.7, 121.3, 127.9, 128.6, 128.7, 129.1, 135.3, 135.9, 156.9, 169.9, 171.8. Elemental Analysis: $\mathrm{C}_{48} \mathrm{H}_{50} \mathrm{O}_{14} \mathrm{~S}_{2} \mathrm{C} 63.01 \%, \mathrm{H} 5.51 \%$. Found C 62.86\%, H 5.39\%.

\section{References}

1. Tanini, D.; Degl'Innocenti, A.; Capperucci, A. Eur. J. Org. Chem. 2015, 357 and references cited therein. http://dx.doi.org/10.1002/ejoc.201403015

2. Capperucci, A.; Tanini, D.; Borgogni, C.; Degl'Innocenti, A. Heteroat. Chem. 2014, $25,678$. http://dx.doi.org/10.1002/hc.21157

3. Tanini, D.; Barchielli, G.; Benelli, F.; Degl'Innocenti, A.; Capperucci, A. Phosphorus, Sulfur, Silicon Relat. Elem. 2015, 190, 1265.

http://dx.doi.org/10.1080/10426507.2014.1002615

4. García Ruano, J. L.; Torrente, E.; Alonso, I.; Rodriguez, M.; Martín-Castro, A. M.; Degl'Innocenti, A.; Frateschi, L.; Capperucci, A. J. Org. Chem. 2012, 77, 1974.

http://dx.doi.org/10.1021/jo202611v

5. Capperucci, A.; Degl'Innocenti, A.; Tiberi, C. Synlett 2011, 2248.

http://dx.doi.org/10.1055/s-0030-1261195

6. Menichetti, S.; Capperucci, A.; Tanini, D.; Braga, A. L.; Botteselle, G. V.; Viglianisi, C. Eur. J. Org. Chem. 2016, 3097.

http://dx.doi.org/10.1002/ejoc.201600351

7. Vazquez-Prieto, M. A.; Miatello, R. M. Mol. Aspects Med. 2010, 31, 540 and references cited therein. http://dx.doi.org/10.1016/j.mam.2010.09.009

8. Wirth, T. Organoselenium Chemistry. Synthesis and Reactions; Wiley-VCH Verlag \& Co, Weinheim, Germany, 2012 and references cited therein.

9. Tanini, D.; Panzella, L.; Amorati, R.; Capperucci, A.; Napolitano, A.; Menichetti, S.; d'Ischia, M. Org. Biomol. Chem. 2015, 5757.

http://dx.doi.org/10.1039/C50B00193E

10. Carretti, E.; Mazzini, V.; Fratini, E.; Ambrosi, M.; Dei, L.; Baglioni, P.; Lo Nostro, P. Phys. Chem. Chem. Phys. 2016, 18, 8865 and references cited therein.

http://dx.doi.org/10.1039/C5CP07792C

11. Venturini, C.; Pomposi, C.; Carretti, E.; Fratini, E.; Lo Nostro, P.; Baglioni, P. J. Phys. Chem. B 2014, 118, 3053.

http://dx.doi.org/10.1021/jp412127y

12. Dolle, C.; Marangone, P.; Riva, S.; Ambrosi, M., Fratini, E.; Peruzzi, N.; Lo Nostro, P. J. Phys. Chem. B 2011, 115, 11638. 
http://dx.doi.org/10.1021/ip204920y

13. Lo Nostro, P.; Capuzzi, G.; Romani, A.; Mulinacci, N. Langmuir 2000, 16, 1744. http://dx.doi.org/10.1021/la990796j

14. Palma, S.; Lo Nostro, P.; Manzo, R.; Allemandi, D. Eur. J. Pharm. Sci. 2002, $16,37$. http://dx.doi.org/10.1016/S0928-0987(02)00054-4

15. Lo Nostro, P.; Ninham, B.W.; Fratoni, L.; Palma, S.; Manzo, R.H.; Allemandi, D.; Baglioni, P. Langmuir 2003, 19, 3222.

http://dx.doi.org/10.1021/la026807h

16. Ambrosi, M.; Lo Nostro, P.; Fratoni, L.; Dei, L.; Ninham, B.W.; Palma, S.; Manzo, R.H.; Allemandi, D.; Baglioni, P. Phys. Chem. Chem. Phys. 2004, 6, 1401.

http://dx.doi.org/10.1039/B314061J

17. Ambrosi, M.; Lo Nostro, P.; Fratini, E.; Giustini, L.; Ninham, B. W.; Baglioni, P. J. Phys. Chem. B 2009, 113, 1404-1412.

http://dx.doi.org/10.1021/jp8092644

18. Mottola, M.; Vico, R. V.; Villanueva, M. E.; Fanani, M. L. J. Colloid Interface Sci. 2015, 45, 232 and references cited therein.

http://dx.doi.org/10.1016/j.jcis.2015.07.014

19. Thopate, S.R.; Rohit A. Dengale, R.A.; Kulkarni, M.G. Synlett 2013, 1555.

http://dx.doi.org/10.1055/s-0033-1338858

20. Karmee, S. K. Lipid Technol. 2011, 23, 227.

http://dx.doi.org/10.1002/lite.201100146

21. Tripathi, R. P.; Singh, B.; Singh Bisht, S.; Pandey, J. Curr. Org. Chem. 2009, 13, 99 and references cited therein.

http://dx.doi.org/10.2174/138527209787193792

22. Karmee, S. K. Appl. Microbiol. Biotechnol. 2009, 81, 1013.

http://dx.doi.org/10.1007/s00253-008-1781-y

23. Capuzzi, G.; Lo Nostro, P.; Kulkarni, K.; Fernandez, J. E. Langmuir 1996, 12, 3957 and references cited.

24. Yoichi Ihori, Y.; Inoue, S.; Fujii, T.; Osawa, Y.; Tanaka, T.; Ikeuchi, H. PCT Int. Appl. WO 2014050894, 2014; SciFinder Scholar 2014:528792.

25. Choi, H.-I.; Kim, H.-J.; Park, J.-I.; Shin, E.-H.; Kim, D.-W.; Kim, S.-S. Bioorg. Med. Chem. Lett. 2009, 19, 2079.

http://dx.doi.org/10.1016/j.bmcl.2008.10.112

26. Zhuang, D.; Nie, J.; Yang, J J. Polym. Sci., Part A: Polym. Chem. 2011, 49, 1999. http://dx.doi.org/10.1002/pola.24627

27. Alam, M., Khan, M. H. J. Pharm. (Lahore) 1979, 1, 67.

28. Balen, M.; Silveira, C.; Kratz, J. M.; Simões, C.M.O.; Valério, A.; Ninow, J.L.; Nandi, L. G.; Di Luccio, M.; de Oliveira, D. Biocatal. Agric. Biotechnol. 2015, 4, 514 and references cited therein.

29. Yan, R.; Jiang, C.; Li, Z.; Liu, L.; Jiang, X., Pat. Appl. CN 104480156 A, 2015; SciFinder Scholar 2015:567544.

30. Stojanović, M.; Velićković, D.; Dimitrijević, A.; Milosavić, N.; Knežević-Jugović, Z.; Bezbradica, D. J. Oleo Sci. 2013, 62, 591.

http://dx.doi.org/10.5650/jos.62.591

31. Moreno-Perez, S.; Filice, M.; Guisan, J.M.; Fernandez-Lorente, G. Chem. Phys. Lipids 2013, $174,48$. http://dx.doi.org/10.1016/i.chemphyslip.2013.06.003 
32. Reyes-Duarte, D.; Lopez-Cortes, N.; Torres, P.; Comelles, F.; Parra, J. L.; Peña, S.; Ugidos, A. V.; Ballesteros, A.; Plou, F. J. J. Am. Oil Chem. Soc. 2011, 88, 57.

http://dx.doi.org/10.1007/s11746-010-1643-5

33. Kaiyal, M. U.S. Pat. Appl. US 20110151525 A1, 2011; SciFinder Scholar 2011:787884.

34. Adamczak, M.; Bornscheuer, U. T. Process Biochem. 2009, $44, \quad 257$. http://dx.doi.org/10.1016/j.procbio.2008.10.014

35. Kim, J. D.; Han, G.; Zee, O. P.; Jung, Y. H. Tetrahedron Lett. 2003, 44, 733. http://dx.doi.org/10.1016/S0040-4039(02)02648-5

36. Jung, M. E.; Koch, P. Tetrahedron Lett. 2011, 52, 6051.

http://dx.doi.org/10.1016/j.tetlet.2011.08.102

37. Yu, W.; Su, M.; Gao, X.; Yang, Z.; Jin, Z. Tetrahedron Lett. 2000, 41, 4015.

http://dx.doi.org/10.1016/S0040-4039(00)00574-8

38. Máté Kicsák, M.; Bege, M.; Bereczki, I.; Csávás, M.; Herczeg, M.; Kupihár, Z.; Kovács, L.; Borbás, A.; Herczegh, P. Org. Biomol. Chem. 2016, 14, 3190 and references cited therein. http://dx.doi.org/10.1039/C60B00067C

39. Suzuki, Y.; Saitoh, G.; Nawa, S.; Kodomari, M. Nippon Kagaku Kaishi 1998, $12,824$. http://dx.doi.org/10.1246/nikkashi.1998.824

40. Goupy, P.; Dufour, C.; Loonis, M.; Dangles, O. J. Agric. Food Chem., 2003, 51, 615. http://dx.doi.org/10.1021/jf025938l

41. Ravindar, K., Caron, P-I., Deslongchamps, P. J. Org. Chem., 2014, 79, 17, 7979. 